\title{
IRREVERSIBILITY AND DISSIPATION IN NEUTRAL B-MESON DECAYS
}

\author{
F. Benatti \\ Dipartimento di Fisica Teorica, Università di Trieste \\ Strada Costiera 11, 34014 Trieste, Italy \\ and \\ Istituto Nazionale di Fisica Nucleare, Sezione di Trieste \\ R. Floreanini \\ Istituto Nazionale di Fisica Nucleare, Sezione di Trieste \\ Dipartimento di Fisica Teorica, Università di Trieste \\ Strada Costiera 11, 34014 Trieste, Italy \\ R. Romano \\ Dipartimento di Fisica Teorica, Università di Trieste \\ Strada Costiera 11, 34014 Trieste, Italy \\ and \\ Istituto Nazionale di Fisica Nucleare, Sezione di Trieste
}

\begin{abstract}
The propagation and decay of neutral $B$-mesons can be described in terms of quantum dynamical semigroups; they provide generalized timeevolutions that take into account possible non-standard effects leading to loss of phase coherence and dissipation. These effects can be fully parametrized in terms of six phenomenological constants. A detailed analysis of selected $B$-meson decays shows that present and future dedicated experiments, both at colliders and $B$-factories, will be able to put stringent bounds on these non-standard parameters.
\end{abstract}




\section{INTRODUCTION}

The study of physics related to $b$-flavored hadrons appears to be a very promising testing ground for various fundamental aspects of the standard model. Indeed, a considerable effort has recently been devoted to the realization of specific experimental programs in order to probe with high accuracy the $b$-sector of the theory. The so-called $B$-factories are already collecting data, while experiments at colliders will start running in the near future and other will be constructed in the coming years, so that very precise data, in particular on $C P$-violating phenomena, will be available for comparison with the theoretical predictions.[1-3]

The neutral $B$-meson system is in this respect a unique laboratory: the $B^{0}-\overline{B^{0}}$ mixing and the induced interference phenomena allow, at least in principle, an accurate analysis of very small effects. Besides $C P$-violating phenomena, these could also include effects induced by physics beyond the standard model, like those predicted by gran-unified theories, supersymmetry and even fundamental dynamics of strings and branes.

In the following, we shall devote our attention to the study of the non-standard effects that are induced in neutral $B$-meson physics by a suitable generalization of the familiar, effective time-evolution of ordinary quantum mechanics. This generalized dynamics takes into account possible phenomena leading to irreversibility and dissipation that could affect various $B$-meson observables; we shall discuss in detail to what extent present and future dedicated experiments will be able to detect such effects.

Our approach will be phenomenological in nature; in particular, we shall not need to discuss in detail how the generalized time-evolution originates from the fundamental dynamics. In fact, the effective description we shall discuss turns out to be largely independent from the microscopic phenomena responsible for the appearance of the new effects.

This phenomenological point of view is perfectly consistent: not all dynamics that generalize the familiar, unitary time-evolution of quantum mechanics are in fact physically acceptable; basic requirements need to be enforced. The new time-evolution should always transform, in all physical situations, $B$-meson states into $B$-meson states, allow forward in time composition law, while increasing the system entropy. As we shall see, these conditions uniquely fix the form of the generalized dynamics, that turns out to be parametrized in terms of six, new phenomenological constants. In more mathematically precise terms, the generalized time-evolution takes the form of a quantum dynamical semigroup.[4-6]

A physical instance in which evolutions of this type are encountered is given by the study of open quantum systems. [4-8] Quite in general, an open system can be considered as a subsystem $S$ in interaction with a large environment $E$. Although the complete system $S+E$ evolves in time with the standard unitary operator of quantum mechanics, the subdynamics of $S$ alone, obtained by a suitable integration over the environment degrees of freedom, is rather involved, showing in general irreversibility and memory effects. However, when the interaction between the subsystem and the environment can be considered to be weak, the dynamics of $S$ simplifies; it can be represented by linear maps, local in time, that take precisely the form of a quantum dynamical semigroup.

This description is very general and can be applied to model different physical situa-

tions. It has been originally developed in the framework of quantum optics, [8-10] but it has 
also been successfully used to study statistical models, [4-6] the interaction of a microsystem with a measuring apparatus, [11-13] non-standard effects in different interferometric experiments involving elementary particles (neutrons, [14] neutrinos [15] and photons [16]). ${ }^{\dagger}$ Furthermore, it provides a general framework for the analysis of dissipative effects in the evolution and decay of neutral meson systems.[19, 20, 21-25] (For early investigations, see [26].)

The original physical motivation for such investigations came from quantum gravity: due to the quantum fluctuation of the gravitational field and the presence of virtual black holes, space-time should lose its continuum character at Planck's scale, and this could lead to loss of quantum coherence.[26-33] From a more fundamental point of view, also the dynamics of strings and branes could act as an effective environment, inducing nonstandard, dissipative effects at low energies. [34, 35] Fortunately, as already mentioned, the details of the microscopic, "stringy" dynamics are not needed for the effective description of the dissipative phenomena in terms of quantum dynamical semigroups. [35] In this respect, this phenomenological approach provides a universal framework for the study of quantum decoherence effects.

In the case of neutral kaon system, the analysis of the dissipative phenomena has been pursued in detail, and upper bounds on some of the constants parametrizing the new effects have been obtained using available experimental data. [21, 22] Improvements of these results are expected from the experimental study of correlated $K$-mesons at $\phi$-factories. $[23,24]$

For the case of the neutral $B$-meson system, a preliminary analysis of the relevance of dissipative effects on semileptonic decays has been presented in [25]. There, a particular phase choice for the $B$ states has been adopted; it allowed treating indirect $C P$-violating effects on the same footing as the decoherence phenomena.

In the following, a much more general and complete discussion will be presented. Using a manifestly phase-invariant formalism that makes no commitment on the magnitude of $C P$ (and $C P T$ ) violating effects, the impact of the irreversible, dissipative phenomena on neutral $B$-meson decays will be analyzed and discussed in detail, using both semileptonic and selected hadronic decay channels. We shall first study decays of single mesons, relevant for experiments at colliders, and then discuss the case of correlated mesons at $B$-factories. In both cases, the new, dissipative phenomena modify in a specific and characteristic way the various $B$-meson observables, so that the presence of dissipation can be probed quite independently from other non-standard effects. ${ }^{\ddagger}$ Although more specific studies, that include precise analysis of acceptance and efficiency of the various detectors, are certainly necessary, our investigation clearly indicates that present and future $B$-meson experiments should be able to put stringent limits on the parameters that describe nonstandard, dissipative phenomena.

$\dagger$ Propagation of neutrinos in thermal environments can also be described by equations of semigroup form; for details, see [17, 18] and the discussion in [15]

$\ddagger$ For recent studies on possible violations of the CPT symmetry and other, unconventional phenomena in $B$-decays, see [36-41] and references therein. 


\section{QUANTUM DYNAMICAL SEMIGROUPS}

The familiar effective description of the propagation and decay of neutral $B$-mesons requires the introduction of a two-dimensional Hilbert space; ${ }^{\dagger}$ the time-evolution is then realized by a linear transformation on the elements of this space.[1, 2] In presence of dissipation however, a more general formalism is needed, in which $B$-meson states are represented by density matrices. These are hermitian, positive operators (i.e. with positive eigenvalues), with constant trace (at least for unitary evolutions). In the $\left|B^{0}\right\rangle,\left|\overline{B^{0}}\right\rangle$ basis, any $B$-meson state can then be written as

$$
\rho=\left[\begin{array}{ll}
\rho_{1} & \rho_{3} \\
\rho_{4} & \rho_{2}
\end{array}\right],
$$

where $\rho_{4} \equiv \rho_{3}^{*}$, and $*$ signifies complex conjugation.

As explained in the introductory remarks, our analysis is based on the hypothesis that the time evolution of the state $\rho$ is realized in terms of linear transformations: they are generated by an equation that extends the familiar quantum mechanical one, being of the form:

$$
\frac{\partial \rho(t)}{\partial t}=-i H \rho(t)+i \rho(t) H^{\dagger}+L[\rho] .
$$

The first two pieces on the r.h.s. give the standard hamiltonian contribution, while $L$ is a linear map that encodes possible dissipative, non-standard effects.

The effective hamiltonian $H$ includes a non-hermitian part,

$$
H=M-\frac{i}{2} \Gamma,
$$

with $M$ and $\Gamma$ hermitian matrices, that characterizes the natural width of the states. The entries of $H$ can be expressed in terms of its eigenvalues: $\lambda_{S}=m_{S}-\frac{i}{2} \gamma_{S}, \lambda_{L}=$ $m_{L}-\frac{i}{2} \gamma_{L}$, and the complex parameters $p_{S}, q_{S}, p_{L}, q_{L}$, appearing in the corresponding (right) eigenstates,

$$
\begin{array}{rlrl}
\left|B_{S}\right\rangle & =p_{S}\left|B^{0}\right\rangle+q_{S}\left|\overline{B^{0}}\right\rangle, & & \left|p_{S}\right|^{2}+\left|q_{S}\right|^{2}=1, \\
\left|B_{L}\right\rangle=p_{L}\left|B^{0}\right\rangle-q_{L}\left|\overline{B^{0}}\right\rangle, & & \left|p_{L}\right|^{2}+\left|q_{L}\right|^{2}=1 .
\end{array}
$$

We use here a notation that follows the conventions usually adopted for the neutral kaon system. The two states in (2.4) are expected to have a negligible width difference,

$$
\Delta \Gamma \ll \Gamma, \quad \Delta \Gamma=\gamma_{S}-\gamma_{L}, \quad \Gamma=\frac{\gamma_{S}+\gamma_{L}}{2},
$$

so that they may be more conveniently distinguished via their mass difference, $\Delta m=$ $m_{L}-m_{S}$, rather than their different lifetimes. For this reason, the notation $\left|B_{L}\right\rangle$, for the

$\dagger$ We shall limit our considerations to $B_{d}$-mesons, although most of the discussions below could be applied to $B_{s}$-mesons as well. 
light meson, and $\left|B_{H}\right\rangle$, for the heavy partner are therefore sometimes used instead of those in $(2.4) .[1,2]$

The effective hamiltonian $H$ can be diagonalized using the similarity transformation induced by (2.4):

$$
H=V H_{0} V^{-1}
$$

with

$$
V=\left[\begin{array}{cc}
p_{S} & p_{L} \\
q_{S} & -q_{L}
\end{array}\right], \quad H_{0}=\left[\begin{array}{cc}
\lambda_{S} & 0 \\
0 & \lambda_{L}
\end{array}\right]
$$

Then, one can write:

$$
H \equiv\left[\begin{array}{cc}
H_{1} & H_{3} \\
H_{4} & H_{2}
\end{array}\right]=\frac{1}{r_{S}+r_{L}}\left[\begin{array}{cc}
r_{S} \lambda_{S}+r_{L} \lambda_{L} & r_{S} r_{L}\left(\lambda_{S}-\lambda_{L}\right) \\
\lambda_{S}-\lambda_{L} & r_{L} \lambda_{S}+r_{S} \lambda_{L}
\end{array}\right]
$$

where we find useful to introduce the two ratios:

$$
r_{S}=\frac{p_{S}}{q_{S}}, \quad r_{L}=\frac{p_{L}}{q_{L}} .
$$

They can be conveniently expressed as

$$
r_{S}=\sqrt{\sigma} \sqrt{\frac{1+\theta}{1-\theta}}, \quad r_{L}=\sqrt{\sigma} \sqrt{\frac{1-\theta}{1+\theta}}
$$

in terms of the two complex parameters

$$
\sigma=r_{S} r_{L}, \quad \theta=\frac{r_{S}-r_{L}}{r_{S}+r_{L}}
$$

that signal $T$ and $C P T$ violating effects. Indeed, from (2.8) one has:

$$
\sigma=\frac{H_{3}}{H_{4}}, \quad \theta=\frac{H_{1}-H_{2}}{\lambda_{S}-\lambda_{L}}
$$

so that $T$ (and $C P$ ) invariance is broken when $|\sigma| \neq 1$, while $C P T$ (and $C P$ ) invariance is lost for $\theta \neq 0 .[1,2]$

Even in absence of the additional piece $L[\rho]$ in (2.2), probability is not conserved during the time evolution: $d \operatorname{Tr}[\rho(t)] / d t \leq 0$. This is due to the presence of a non-hermitian part in the effective hamiltonian $H$. On the other hand, loss of phase coherence shows up only when the piece $L[\rho]$ is nonvanishing: it produces dissipation and possible transitions from pure states to mixed states.

As already mentioned before, not all maps $L[\rho]$ gives rise to integrated time evolutions, $\gamma_{t}: \rho(0) \mapsto \rho(t)$, that are physically acceptable. Quite in general, the one parameter (=time) family of linear maps $\gamma_{t}$ should transform $B$-meson states into $B$-meson states, and therefore should map an initial density matrix into a density matrix; further, it should have the property of increasing the (von Neumann) entropy, $S=-\operatorname{Tr}[\rho(t) \ln \rho(t)]$, of obeying the semigroup composition law, $\gamma_{t}\left[\rho\left(t^{\prime}\right)\right]=\rho\left(t+t^{\prime}\right)$, for $t, t^{\prime} \geq 0$, of preserving the 
positivity of $\rho(t)$ for all times. Actually, for the physical consistency of the formalism in the case of correlated systems, one has to demand that the time evolution $\gamma_{t}$ be completely positive.[4-6] Once these properties are taken into account, the form of $L[\rho]$ results uniquely fixed and the family of maps $\gamma_{t}$ represents a so-called quantum dynamical semigroup.

The linear map $L[\rho]$ can be fully parametrized in terms of six real phenomenological constants, $a, b, c, \alpha, \beta$ and $\gamma$, with $a, \alpha, \gamma$ non negative, satisfying the following inequalities: $[21,23]$

$$
\begin{array}{lr}
2 R \equiv \alpha+\gamma-a \geq 0, & R S-b^{2} \geq 0, \\
2 S \equiv a+\gamma-\alpha \geq 0, & R T-c^{2} \geq 0, \\
2 T \equiv a+\alpha-\gamma \geq 0, & S T-\beta^{2} \geq 0, \\
R S T-2 b c \beta-R \beta^{2}-S c^{2}-T b^{2} \geq 0,
\end{array}
$$

direct consequence of the property of complete positivity. A convenient explicit expression for $L[\rho]$ can be obtained by introducing a vector notation for the matrix $\rho$, rewritten as a four-dimensional column vector $|\rho\rangle$, with components $\left(\rho_{1}, \rho_{2}, \rho_{3}, \rho_{4}\right)$. In this way, the map $L[\rho]$ can be represented by a $4 \times 4$ hermitian matrix $\mathcal{L}$, acting on $|\rho\rangle$ :

$$
\mathcal{L}=\left[\begin{array}{cccc}
-a & a & -(c-i b) & -(c+i b) \\
a & -a & c-i b & c+i b \\
-(c+i b) & c+i b & -(\alpha+\gamma) & \alpha-\gamma-2 i \beta \\
-(c-i b) & c-i b & \alpha-\gamma+2 i \beta & -(\alpha+\gamma)
\end{array}\right]
$$

Among the physical requirements that the complete time evolution $\gamma_{t}$ should satisfy, complete positivity is perhaps the less intuitive. It is often dismissed, in favor of the more obvious simple positivity. Simple positivity is in fact generally enough to guarantee that the eigenvalues of the density matrix $\rho(t)$ remain positive at any time; this requirement is obviously crucial for the consistency of the formalism, in view of the interpretation of the eigenvalues of $\rho(t)$ as probabilities.

Complete positivity is a stronger property, in the sense that it assures the positivity of the density matrix describing the states of a larger system, involving the coupling with an extra, auxiliary finite-dimensional system. Although trivially satisfied by standard quantum mechanical (unitary) time-evolutions, the requirement of complete positivity seems at first a mere technical complication. Nevertheless, it turns out to be essential in properly treating correlated systems, like the two $B$-meson coming from the $\Upsilon(4 S)$ resonance; [42] it assures the absence of unphysical effects, like the appearance of negative probabilities, that could occur for just simply positive dynamics (see the discussion in Sect.7).

A further comment on the expression (2.14) of the dissipative part of the evolution equation (2.2) is in order. The entries of the matrix (2.14) have been written in a specific choice of basis in the two-dimensional Hilbert space, the one for which the state $\rho$ takes the form (2.1). If one performs a (unitary) transformation on the basis vectors $\left|B^{0}\right\rangle,\left|\overline{B^{0}}\right\rangle$, also the six parameters appearing in (2.14) will in general change to new ones $a^{\prime}, b^{\prime}, c^{\prime}, \alpha^{\prime}, \beta^{\prime}$, and $\gamma^{\prime}$, expressed as linear combinations of the old ones. Despite this, one can check that 
the inequalities in (2.13) are form-invariant; in other terms, the transformed dissipative parameters still obey (2.13), provided the old ones do. ${ }^{\dagger}$

This discussion clearly illustrates that, although the equation (2.2) has been written in a fixed, specific $B$-meson basis, the notion of complete positivity is basis-independent, as is the description of the dissipative effects: if the contribution $\mathcal{L}$ in $(2.14)$ is non vanishing in one basis, it is non vanishing in any basis. This result has to be expected from the general theory of quantum dynamical semigroups, that can be formulated in a basis-independent way, [4-6] and will be made apparent in the discussion of the coming sections.

\section{THE EFFECTIVE TIME-EVOLUTION}

The behaviour in time of physical observables related to the various $B$-meson decay channels can be obtained by solving the evolution equation (2.2) for an arbitrary initial state $\rho(0)$. This results in the study of a system of linear differential equations for the entries of the density matrix $\rho$ in (2.1).

It is convenient to use the vector notation introduced in the previous section, and write the matrix $\rho$ as the four-dimensional vector $|\rho\rangle$. Then, the evolution equation (2.2) takes the form of a Schrödinger (or diffusion) equation:

$$
\frac{d}{d t}|\rho(t)\rangle=\mathcal{K}|\rho(t)\rangle \equiv[\mathcal{H}+\mathcal{L}]|\rho(t)\rangle
$$

where $\mathcal{H}$ is the $4 \times 4$ matrix containing the hamiltonian contributions $[c f .(2.8)]$,

$$
\mathcal{H}=\left[\begin{array}{cccc}
2 \mathcal{I} m\left(H_{1}\right) & 0 & i H_{3}^{*} & -i H_{3} \\
0 & 2 \mathcal{I} m\left(H_{2}\right) & -i H_{4} & i H_{4}^{*} \\
i H_{4}^{*} & -i H_{3} & i\left(H_{2}^{*}-H_{1}\right) & 0 \\
-i H_{4} & i H_{3}^{*} & 0 & i\left(H_{1}^{*}-H_{2}\right)
\end{array}\right]
$$

while the dissipative part $\mathcal{L}$ is given in (2.14).

As already mentioned before, the hamiltonian piece in (3.2) contains contributions that are not invariant under $C P T$ and $T$ transformations; this is also true for $\mathcal{L}$, so that in general dissipation will induce violations of these discrete symmetries. It is instructive to explicitly discuss this point in the formalism of $(3.1)$. $^{\ddagger}$

Following the rules of quantum mechanics, any symmetry transformation can be realized by a unitary or antiunitary operator $U$ acting on the basis states $\left|B^{0}\right\rangle,\left|\overline{B^{0}}\right\rangle$, or alternatively on the density matrix $\rho$. This induces an action on the vector $|\rho\rangle$, realized by a $4 \times 4$ matrix $\mathcal{U}$ :

$$
|\rho\rangle \rightarrow\left|\rho^{\prime}\right\rangle=\mathcal{U}|\rho\rangle
$$

$\dagger$ For a generic, non-unitary change of basis, as the one discussed in Appendix A, also the form of the conditions (2.13) in general changes, while always assuring the fulfillment of the property of complete positivity.

$\ddagger$ Further discussions on the notion of symmetry invariance for quantum dynamical semigroups can be found in [43]. 
This transformation leaves the equation (3.1) form-invariant, i.e. it is a symmetry of the evolution equation, provided:

$$
\mathcal{U} \mathcal{K U}^{-1}=\mathcal{K}
$$

for unitary transformations, or alternatively

$$
\mathcal{U} \mathcal{K} \mathcal{U}^{-1}=\mathcal{K}^{\dagger}
$$

in the case of antiunitary transformations.

For instance, an independent phase change of the two basis vectors,

$$
\left|B^{0}\right\rangle \rightarrow e^{i \phi}\left|B^{0}\right\rangle, \quad\left|\overline{B^{0}}\right\rangle \rightarrow e^{i \bar{\phi}}\left|\overline{B^{0}}\right\rangle
$$

is realized by the $4 \times 4$ diagonal matrix:

$$
\mathcal{U}_{\phi}=\operatorname{diag}\left[1,1, e^{i(\phi-\bar{\phi})}, e^{-i(\phi-\bar{\phi})}\right] .
$$

One easily checks that this transformation is not an invariance of $\mathcal{H}$ and hence of (3.1); indeed, the off-diagonal elements $H_{3}$ and $H_{4}$ of the effective hamiltonian $H$ do change under (3.5). As a consequence neither $\sigma$ in (2.12), nor $r_{S}$ and $r_{L}$ are phase invariant.

In the case of discrete $C P T, T$ and $C P$ transformations, one finds that the corresponding matrix $\mathcal{U}$ takes a block diagonal form: explicitly, one finds:

$$
\mathcal{U}_{C P T}=\left[\begin{array}{cc}
\sigma_{1} & 0 \\
0 & \sigma_{0}
\end{array}\right], \quad \mathcal{U}_{T}=\left[\begin{array}{cc}
\sigma_{0} & 0 \\
0 & \sigma_{\varphi}
\end{array}\right], \quad \mathcal{U}_{C P}=\left[\begin{array}{cc}
\sigma_{1} & 0 \\
0 & \sigma_{\varphi}
\end{array}\right],
$$

where $\sigma_{i}, i=1,2,3$ represent the usual Pauli matrices, $\sigma_{0}$ being the identity, while

$$
\sigma_{\varphi}=\left[\begin{array}{cc}
0 & e^{-2 i \varphi} \\
e^{2 i \varphi} & 0
\end{array}\right]
$$

contains the dependence on the phase that defines the $C P$ transformation of the $B^{0}-\overline{B^{0}}$ basis states: $\left|B^{0}\right\rangle \rightarrow e^{i \varphi}\left|\overline{B^{0}}\right\rangle$.

For the hamiltonian contribution $\mathcal{H}$, insertion of the expressions (3.7) in the appropriate invariance condition (3.4) gives the familiar results. In particular, $C P T$ invariance requires $H_{1}=H_{2}$, or equivalently, recalling the definitions (2.11), (2.12):

$$
r_{S}=r_{L}, \quad \theta=0
$$

while $T$ invariance implies $\left|H_{3}\right|=\left|H_{4}\right|$, i.e.

$$
\left|r_{S} r_{L}\right|=1, \quad|\sigma| \equiv \frac{1+\xi}{1-\xi}=1, \quad \xi=0
$$

Further, notice that imposing both $C P T$ and $T$ invariance, hence $C P$ invariance, i.e. $\theta=\xi=0$, readily implies:

$$
r_{S}=r_{L}=\sqrt{\sigma}, \quad \sigma=e^{-2 i \varphi}
$$


For the dissipative part $\mathcal{L}$ of the pseudo hamiltonian $\mathcal{K}$ similar invariant conditions can be derived using (3.4a) (the matrix in (2.14) is in fact hermitian); they impose constraints on the six dissipative parameters $a, b, c, \alpha, \beta$ and $\gamma$. More specifically, invariance under $C P T$-transformations requires the vanishing of $c$ and $b$, while $T$-invariance imposes the conditions: $(c-i b)=e^{2 i \varphi}(c+i b)$ and $(\alpha-\gamma+2 i \beta)=e^{4 i \varphi}(\alpha-\gamma-2 i \beta)$. Finally, $C P$ invariance requires $(c-i b)=-e^{2 i \varphi}(c+i b)$ and $(\alpha-\gamma+2 i \beta)=e^{4 i \varphi}(\alpha-\gamma-2 i \beta)$. It should be noticed that all these constraints are perfectly compatible with the inequalities in (2.13); in other words, complete positivity does not interfere with the discrete transformations.

Although the available experimental bounds on the $C P T$ and $T$ violating contributions of the hamiltonian $\mathcal{H}$ are not very accurate, the magnitude of the constants $\theta$ and $\xi$ parametrizing these violations are expected to be very small. Despite this, in the discussion that follows we shall try to be as general as possible, and keep $\theta$ and $\xi$, hence $r_{S}$ and $r_{L}$, arbitrary, unless explicitly stated.

In solving the evolution equation (3.1) however, we shall assume the dissipative contribution $\mathcal{L}$ to be small. In a phenomenological approach, it is hard to give an apriori estimate on how large the dissipative effects should be. However, as already mentioned in the introductory remarks, a general framework in which dissipation naturally emerges is provided by the study of subsystems in interaction with large environments. In such instances, the non-standard effects can be roughly estimated to be proportional to powers of the typical energy of the system, while suppressed by inverse powers of the characteristic energy scale of the environment.[4-7]

In the case of the $B^{0}-\overline{B^{0}}$ system, these considerations, together with the general idea that dissipation is induced by quantum effects at a large, fundamental scale $M_{F}$, lead to predict very small values for the parameters $a, b, c, \alpha, \beta$ and $\gamma$; using dimensional arguments, an upper bound on the magnitude of these parameters can be roughly evaluated to be of order $m_{B}^{2} / M_{F}$, where $m_{B}$ is the neutral $B$-meson mass. If the non-standard effects have a gravitational origin, the scale $M_{F}$ should coincide with the Planck mass $M_{P}$, and the previous upper bound would give: $m_{B}^{2} / M_{P} \sim 10^{-18} \mathrm{GeV}$.

These considerations allow treating the dissipative piece $\mathcal{L}$ in (3.1) as a perturbation to the hamiltonian contribution $\mathcal{H}$. In order to set up the perturbative expansion, it is useful to make a change of basis in (3.1), so that the hamiltonian piece $\mathcal{H}$ becomes diagonal.

As discussed in the previous section, the effective hamiltonian $H$ can be brought to diagonal form by the similarity transformation (2.6); the diagonalizing matrix $V$ in $(2.7)$ can be conveniently decomposed as:

$$
V=\widetilde{V} \cdot Q, \quad \widetilde{V}=\left[\begin{array}{cc}
r_{S} & r_{L} \\
1 & -1
\end{array}\right], \quad Q=\left[\begin{array}{cc}
q_{S} & 0 \\
0 & q_{L}
\end{array}\right] .
$$

Since the matrix $Q$ is diagonal, it disappears from the relation (2.6), that can be equally well be written as:

$$
H=\widetilde{V} H_{0} \widetilde{V}^{-1} .
$$

When applied to the density matrix $\rho$, this change of basis induces the transformation $\rho \rightarrow \tilde{\rho}=\widetilde{V}^{-1} \rho \widetilde{V}^{\dagger-1}$, or equivalently:

$$
|\rho\rangle \rightarrow|\tilde{\rho}\rangle=\mathcal{V}|\rho\rangle
$$


where

$$
\mathcal{V}=\frac{1}{\left|r_{S}+r_{L}\right|^{2}}\left[\begin{array}{rrrr}
1 & \left|r_{L}\right|^{2} & r_{L}^{*} & r_{L} \\
1 & \left|r_{S}\right|^{2} & -r_{S}^{*} & -r_{S} \\
1 & -r_{S}^{*} r_{L} & -r_{S}^{*} & r_{L} \\
1 & -r_{S} r_{L}^{*} & r_{L}^{*} & -r_{S}
\end{array}\right]
$$

Then, the evolution equation (3.1) becomes

$$
\frac{d}{d t}|\tilde{\rho}(t)\rangle=\left[\mathcal{H}_{0}+\widetilde{\mathcal{L}}\right]|\tilde{\rho}(t)\rangle
$$

with

$$
\widetilde{\mathcal{L}}=\mathcal{V} \mathcal{L} \mathcal{V}^{-1}
$$

and a diagonal hamiltonian piece:

$$
\begin{gathered}
\mathcal{H}_{0}=\left[\begin{array}{cccc}
-\gamma_{S} & 0 & 0 & 0 \\
0 & -\gamma_{L} & 0 & 0 \\
0 & 0 & -\Gamma_{-} & 0 \\
0 & 0 & 0 & -\Gamma_{+}
\end{array}\right], \\
\Gamma_{ \pm}=\Gamma \pm i \Delta m, \quad \Delta m=m_{L}-m_{S} .
\end{gathered}
$$

The price to pay for this change of basis is a more complicated expression for the matrix $\widetilde{\mathcal{L}}$, representing the dissipative contribution (its explicit form is collected in Appendix A). However, the entries of $\widetilde{\mathcal{L}}$ appear now to be manifestly invariant under the change of phase in (3.5), and therefore, the same is true for the whole evolution equation in (3.16). This is a great advantage since any solution of (3.16), even an approximate one obtained using perturbative methods, will result to be manifestly phase-invariant.

We remark that physical observables, being the result of a trace operation (see below), are by definition independent from any phase convention. Provided a sufficient number of observables are computed, one can consistently re-express a result obtained in a given phase convention, into any other phase choice. However, once a phase choice is adopted and a given approximation used, conclusions drawn from only a limited number of physical observations could lead to incorrect conclusions. It is therefore always preferable at any step to work in a phase-independent framework.

The evolution equation in (3.16) can now be solved by iteration, to any order in the small dissipative parameters $a, b, c, \alpha, \beta$ and $\gamma$. Simple manipulations allow to express the vector $|\tilde{\rho}(t)\rangle$ at time $t$ as the series expansion:

$$
\begin{aligned}
|\tilde{\rho}(t)\rangle=e^{\mathcal{H}_{0} t}|\tilde{\rho}(0)\rangle+\int_{0}^{t} d s e^{\mathcal{H}_{0}(t-s)} \widetilde{\mathcal{L}} e^{\mathcal{H}_{0} s}|\tilde{\rho}(0)\rangle \\
\quad+\int_{0}^{t} d s_{1} \int_{0}^{s_{1}} d s_{2} e^{\mathcal{H}_{0}\left(t-s_{1}\right)} \widetilde{\mathcal{L}} e^{\mathcal{H}_{0}\left(s_{1}-s_{2}\right)} \widetilde{\mathcal{L}} e^{\mathcal{H}_{0} s_{2}}|\tilde{\rho}(0)\rangle+\ldots
\end{aligned}
$$

For the considerations that follow, it will be sufficient to consider terms that are at most linear in the small parameters, thus retaining only the first two terms in the r.h.s. of (3.20). 
This suggests a further simplifying assumption, that allows to write down a more manageable expression for the matrix $\widetilde{\mathcal{L}}$.

As it is apparent from its definition in (3.17), the entries of $\widetilde{\mathcal{L}}$ are linear combinations of $a, b, c, \alpha, \beta$ and $\gamma$, with coefficients that depend on $\theta, \xi$ and the phase of $\sigma$. Since $\theta$ and $\xi$ are themselves expected to be small, they can be safely ignored in those expressions. In other terms, it appears reasonable to neglect $C P T$ and $T$ (hence $C P$ ) violating effects induced by non-vanishing $\theta$ and $\xi$, when these violating phenomena mix with the dissipative effects.

Within this approximation, the matrix $\widetilde{\mathcal{L}}$ takes the form

$$
\widetilde{\mathcal{L}}=\left[\begin{array}{rrrr}
-\widetilde{D} & \widetilde{D} & -\widetilde{C} & -\widetilde{C}^{*} \\
\widetilde{D} & -\widetilde{D} & \widetilde{C} & \widetilde{C}^{*} \\
-\widetilde{C}^{*} & \widetilde{C}^{*} & -\widetilde{A} & \widetilde{B} \\
-\widetilde{C} & \widetilde{C} & \widetilde{B}^{*} & -\widetilde{A}
\end{array}\right]
$$

where

$$
\begin{aligned}
\widetilde{A} & =\frac{1}{2}\{[(\alpha+\gamma)+2 a+\mathcal{R} e[(\alpha-\gamma+2 i \beta) \sigma]\}, \\
\widetilde{B} & =\frac{1}{2}\{[(\alpha+\gamma)-2 a+\mathcal{R} e[(\alpha-\gamma+2 i \beta) \sigma]-4 i \mathcal{I} m[(c-i b) \sqrt{\sigma}]\}, \\
\widetilde{C} & =\frac{1}{2}\{2 \mathcal{R} e[(c-i b) \sqrt{\sigma}]+i \mathcal{I} m[(\alpha-\gamma+2 i \beta) \sigma]\}, \\
\widetilde{D} & =\frac{1}{2}\{(\alpha+\gamma)-\mathcal{R} e[(\alpha-\gamma+2 i \beta) \sigma]\},
\end{aligned}
$$

and $\sigma$ is here the pure phase defined in (3.11).

It is now a matter of a simple computation to perform the integrals in (3.20) and find the time dependence of the components $\tilde{\rho}_{1}(t), \tilde{\rho}_{2}(t), \tilde{\rho}_{3}(t), \tilde{\rho}_{4}(t)$ of the vector $|\tilde{\rho}(t)\rangle$, that give the entries of the density matrix $\tilde{\rho}(t)$. The explicit expressions are collected in Appendix B, and will be used in the next sections to study in detail various neutral $B$-meson decays.

\section{OBSERVABLES}

In the formalism of density matrices, any physical observable of the neutral $B$-meson system is described by a suitable hermitian operator $\mathcal{O}$. Its evolution in time can be obtained by taking the trace with the density matrix $\rho(t)$.

Of particular interest are those observables $\mathcal{O}_{f}$ that are associated with the decay of a $B$-meson into final states $f$. In the $\left|B^{0}\right\rangle,\left|\overline{B^{0}}\right\rangle$ basis, $\mathcal{O}_{f}$ is represented by a $2 \times 2$ matrix,

$$
\mathcal{O}_{f}=\left[\begin{array}{ll}
\mathcal{O}_{1} & \mathcal{O}_{3} \\
\mathcal{O}_{4} & \mathcal{O}_{2}
\end{array}\right]
$$


whose entries can be explicitly written in terms of the two independent decay amplitudes $\mathcal{A}\left(B^{0} \rightarrow f\right)$ and $\mathcal{A}\left(\overline{B^{0}} \rightarrow f\right)$ :

$$
\begin{array}{ll}
\mathcal{O}_{1}=\left|\mathcal{A}\left(B^{0} \rightarrow f\right)\right|^{2}, & \mathcal{O}_{3}=\left[\mathcal{A}\left(B^{0} \rightarrow f\right)\right]^{*} \mathcal{A}\left(\overline{B^{0}} \rightarrow f\right), \\
\mathcal{O}_{2}=\left|\mathcal{A}\left(\overline{B^{0}} \rightarrow f\right)\right|^{2}, & \mathcal{O}_{4}=\mathcal{A}\left(B^{0} \rightarrow f\right)\left[\mathcal{A}\left(\overline{B^{0}} \rightarrow f\right)\right]^{*} .
\end{array}
$$

Being a physical quantity, directly accessible to the experiment, its mean value,

$$
\left\langle\mathcal{O}_{f}\right\rangle \equiv \operatorname{Tr}\left[\mathcal{O}_{f} \rho\right]
$$

is however basis independent; it can be computed in any specific representation. In particular, using the transformation $\widetilde{V}$ introduced in (3.12) in the definition (4.3), the time evolution of $\left\langle\mathcal{O}_{f}\right\rangle$ can be written as

$$
\left\langle\mathcal{O}_{f}\right\rangle(t)=\operatorname{Tr}\left[\widetilde{\mathcal{O}}_{f} \tilde{\rho}(t)\right]=\sum_{i=1}^{4} \widetilde{\mathcal{O}}_{i}^{*} \tilde{\rho}_{i}(t)
$$

where the entries $\widetilde{\mathcal{O}}_{i}$ of the transformed matrix $\widetilde{\mathcal{O}}_{f}=\widetilde{V}^{\dagger} \mathcal{O}_{f} \tilde{V}$ have been labelled as in (4.1). This general formula will be repeatedly used in the coming sections to explicitly compute experimentally relevant decay rates and asymmetries.

In parametrizing $B^{0}-\overline{B^{0}}$ decays, it is customary to introduce the following phaseindependent complex quantities: $[1,2]$

$$
\lambda_{S}^{f}=\frac{q_{S}}{p_{S}} \frac{\mathcal{A}\left(\overline{B^{0}} \rightarrow f\right)}{\mathcal{A}\left(B^{0} \rightarrow f\right)}, \quad \lambda_{L}^{f}=\frac{q_{L}}{p_{L}} \frac{\mathcal{A}\left(\overline{B^{0}} \rightarrow f\right)}{\mathcal{A}\left(B^{0} \rightarrow f\right)} .
$$

They can be used to express the entries of the matrix $\widetilde{\mathcal{O}}_{f}$; explicitly, one finds:

$$
\widetilde{\mathcal{O}}_{f}=\left|\mathcal{A}\left(B^{0} \rightarrow f\right)\right|^{2}\left[\begin{array}{cc}
\left|r_{S}\right|^{2}\left|1+\lambda_{S}^{f}\right|^{2} & r_{S}^{*} r_{L}\left(1+\lambda_{S}^{f}\right)^{*}\left(1-\lambda_{L}^{f}\right) \\
r_{S} r_{L}^{*}\left(1+\lambda_{S}^{f}\right)\left(1-\lambda_{L}^{f}\right)^{*} & \left|r_{L}\right|^{2}\left|1-\lambda_{L}^{f}\right|^{2}
\end{array}\right]
$$

It is sometimes convenient to use instead the parameters:

$$
\mu_{S}^{f}=\frac{p_{S}}{q_{S}} \frac{\mathcal{A}\left(B^{0} \rightarrow f\right)}{\mathcal{A}\left(\overline{B^{0}} \rightarrow f\right)}=\frac{1}{\lambda_{S}^{f}}, \quad \mu_{L}^{f}=\frac{p_{L}}{q_{L}} \frac{\mathcal{A}\left(B^{0} \rightarrow f\right)}{\mathcal{A}\left(\overline{B^{0}} \rightarrow f\right)}=\frac{1}{\lambda_{L}^{f}}
$$

one can then rewrite $(4.6)$ as:

$$
\widetilde{\mathcal{O}}_{f}=\left|\mathcal{A}\left(\overline{B^{0}} \rightarrow f\right)\right|^{2}\left[\begin{array}{cc}
\left|1+\mu_{S}^{f}\right|^{2} & -\left(1+\mu_{S}^{f}\right)^{*}\left(1-\mu_{L}^{f}\right) \\
-\left(1+\mu_{S}^{f}\right)\left(1-\mu_{L}^{f}\right)^{*} & \left|1-\mu_{L}^{f}\right|^{2}
\end{array}\right]
$$

The form (4.6) and (4.8) for the observable $\widetilde{\mathcal{O}}_{f}$ are very general and hold for any final decay state $f$. Simplified expressions can however be derived by looking at specific decay channels. 
Let us first consider decays of the neutral $B$-mesons in semileptonic states $h \ell \nu$, where $h$ stands for any allowed charged hadronic state. We shall be as general as possible, and include in our discussion violations of the $\Delta B=\Delta Q$ rule. The amplitudes for the decay of a $B^{0}$ or $\overline{B^{0}}$ state into $h^{-} \ell^{+} \nu$ and $h^{+} \ell^{-} \bar{\nu}$ can then be parametrized in terms of three complex constants, $x_{h}, y_{h}$ and $z_{h}$, as follows:

$$
\begin{aligned}
& \mathcal{A}\left(B^{0} \rightarrow h^{-} \ell^{+} \nu\right)=\mathcal{M}_{h}\left(1-y_{h}\right), \\
& \mathcal{A}\left(\overline{B^{0}} \rightarrow h^{+} \ell^{-} \bar{\nu}\right)=\mathcal{M}_{h}^{*}\left(1+y_{h}^{*}\right) \\
& \mathcal{A}\left(B^{0} \rightarrow h^{+} \ell^{-} \bar{\nu}\right)=z_{h} \mathcal{A}\left(\overline{B^{0}} \rightarrow h^{+} \ell^{-} \bar{\nu}\right), \\
& \mathcal{A}\left(\overline{B^{0}} \rightarrow h^{-} \ell^{+} \nu\right)=x_{h} \mathcal{A}\left(B^{0} \rightarrow h^{-} \ell^{+} \nu\right),
\end{aligned}
$$

where $\mathcal{M}_{h}$ is a common factor. (Note that sometimes, $[44,1,2]$ the notation $\bar{x}_{h} \equiv z_{h}^{*}$ is used instead of $z_{h}$.) The $\Delta B=\Delta Q$ rule would forbid the decays $B^{0} \rightarrow h^{+} \ell^{-} \bar{\nu}$ and $\overline{B^{0}} \rightarrow h^{-} \ell^{+} \nu$, so that the parameters $x_{h}$ and $z_{h}$ measure the violations of this rule. Instead, $C P T$-invariance in the decay process would require $y_{h}=0$. In view of this, the quantities $x_{h}, y_{h}$ and $z_{h}$ are expected to be very small. In the computation of semileptonic observables, it seems therefore justified to keep only first order terms in these parameters, neglecting terms that contain $x_{h}, y_{h}$ and $z_{h}$ multiplied by the dissipative parameters or the constants $\theta$ and $\xi$, that signal $C P T$ and $T$ violations in "mixing". Within this approximation, the quantities in (4.5) and (4.7) reduce to

$$
\lambda_{S}^{h^{-}}=\lambda_{L}^{h^{-}}=\sqrt{\sigma^{*}} x_{h} \equiv \lambda_{h}, \quad \mu_{S}^{h^{+}}=\mu_{L}^{h^{+}}=\sqrt{\sigma} z_{h} \equiv \mu_{h},
$$

where $\sigma$ is the pure phase of (3.11).

For hadronic final states, such simplifications are in general not possible. Nevertheless, to be consistent with the approximation adopted in the previous section, when the coefficients $\lambda_{S}, \lambda_{L}, \mu_{S}$ and $\mu_{L}$ multiply a dissipative parameter, they should be computed in the limit of exact $C P T$ and $C P$ symmetries. This is particularly relevant when the final state $f$ has a definite $C P$-parity $\zeta_{f}$; within that approximation, one in fact finds:[1]

$$
\lambda_{S}^{f}=\lambda_{L}^{f}=\zeta_{f}
$$

As we will see in the following, this observation will be helpful in the explicit evaluation of the asymmetries involving decays into these specific final states.

\section{SINGLE MESON DECAYS}

In this section, we shall study observables connected to the time-evolution and decay of a single, uncorrelated $B^{0}-\overline{B^{0}}$ system, that can be typically measured at colliders. Let us indicate with $\left|\tilde{\rho}_{B^{0}}(t)\right\rangle,\left|\tilde{\rho}_{\bar{B}^{0}}(t)\right\rangle$ the time evolution according to (3.16) of the states $\left|\tilde{\rho}_{B^{0}}\right\rangle$, $\left|\tilde{\rho}_{\bar{B}^{0}}\right\rangle$ that represent initial pure $B^{0}, \overline{B^{0}}$ mesons; their components can be organized in the two matrices:

$$
\tilde{\rho}_{B^{0}}=\frac{1}{\left|r_{S}+r_{L}\right|^{2}}\left[\begin{array}{ll}
1 & 1 \\
1 & 1
\end{array}\right], \quad \tilde{\rho}_{\bar{B}^{0}}=\frac{1}{\left|r_{S}+r_{L}\right|^{2}}\left[\begin{array}{cc}
\left|r_{L}\right|^{2} & -r_{S}^{*} r_{L} \\
-r_{S} r_{L}^{*} & \left|r_{S}\right|^{2}
\end{array}\right] .
$$


As discussed in the previous section, the probability rate that an initial $\left|\tilde{\rho}_{B^{0}}\right\rangle,\left|\tilde{\rho}_{\bar{B}^{0}}\right\rangle$ state decays at time $t$ into a given final state $f$ described by the operator $\mathcal{O}_{f}$ is given by:

$$
\mathcal{P}_{f}\left(B^{0} ; t\right)=\operatorname{Tr}\left[\widetilde{\mathcal{O}}_{f} \tilde{\rho}_{B^{0}}(t)\right], \quad \mathcal{P}_{f}\left(\overline{B^{0}} ; t\right)=\operatorname{Tr}\left[\widetilde{\mathcal{O}}_{f} \tilde{\rho}_{\bar{B}^{0}}(t)\right]
$$

In writing down the explicit form of the probabilities $\mathcal{P}$, it is convenient to introduce the new variable $\tau=t \Gamma$; in practice, $\tau$ expresses the time variable in units of the $B$ lifetime. It is also customary to define the two combinations: ${ }^{\dagger}$

$$
\omega=\frac{\Delta m}{\Gamma}, \quad \delta=\frac{\Delta \Gamma}{2 \Gamma} .
$$

Although not yet directly measured, $\delta$ is expected to be very small, $\delta \leq 10^{-2}$. For this reason, in discussing $B^{0}-\overline{B^{0}}$ observables, one often takes the simplified assumption $\delta=0$. This choice produces however important consequences. Indeed, in the limit $\Delta \Gamma=0$, the antihermitian part $\Gamma$ of the effective hamiltonian $H$ in (2.3) becomes proportional to the identity. In this case, the similarity transformation (2.6) that diagonalizes $H$ turns out to be unitary and the eigenstates $\left|B_{S}\right\rangle$ and $\left|B_{L}\right\rangle$ in (2.4) become orthogonal. Since, $\left\langle B_{L} \mid B_{S}\right\rangle=p_{S} p_{L}^{*}-q_{S} q_{L}^{*}$, this implies the condition

$$
r_{S} r_{L}^{*}=1
$$

and as a consequence, $|\sigma|=1$, or equivalently $\xi=0$, and also $\mathcal{I} m(\theta)=0$. Therefore, the condition $\delta=0$ implies $T$ conservation in the hamiltonian piece of the evolution equation, while $C P T$ violation is possible only if $\mathcal{R} e(\theta)$ is different from zero. This fact has clearly important consequences for experimental tests of $C P T$ and $T$ invariance in "mixing". $[45,46]$ In the following, we shall keep $\delta$ nonvanishing, unless explicitly stated.

We shall first consider observables connected with $B$-meson decays into semileptonic final states. Using the appropriate observables (4.6), (4.8) and the results in (4.10) together with those collected in Appendix B, expressions for the probabilities (5.2) can be explicitly obtained. Apart from a common exponential decay factor, they show an oscillatory behaviour modulated by $\omega$ and further exponential terms regulated by $\delta$ :

$$
\begin{aligned}
\mathcal{P}_{h^{-}}\left(B^{0} ; \tau\right)=\frac{\left|\mathcal{M}_{h}\right|^{2}}{2} e^{-\tau}\left\{\cos \omega \tau\left[\frac{4 \mathcal{R} e\left(r_{S} r_{L}^{*}\right)}{\left|r_{S}+r_{L}\right|^{2}} e^{-(A-D) \tau}-2 \mathcal{R} e\left(y_{h}\right)-\frac{4 \delta}{\delta^{2}+\omega^{2}} \mathcal{R} e(C)\right]\right. \\
+\sin \omega \tau\left[-\frac{4 \mathcal{I} m\left(r_{S} r_{L}^{*}\right)}{\left|r_{S}+r_{L}\right|^{2}}-2 \mathcal{I} m\left(\lambda_{h}\right)+\mathcal{R} e(B)\right] \\
+\cosh \delta \tau\left[\frac{2\left(\left|r_{S}\right|^{2}+\left|r_{L}\right|^{2}\right)}{\left|r_{S}+r_{L}\right|^{2}}-2 \mathcal{R} e\left(y_{h}\right)+\frac{4 \delta}{\delta^{2}+\omega^{2}} \mathcal{R} e(C)\right] \\
\left.+\sinh \delta \tau\left[-\frac{2\left(\left|r_{S}\right|^{2}-\left|r_{L}\right|^{2}\right)}{\left|r_{S}+r_{L}\right|^{2}}-2 \mathcal{R} e\left(\lambda_{h}\right)+\frac{D}{\delta}\right]\right\},
\end{aligned}
$$

$\dagger$ They are usually called $x_{B}$ and $y_{B}$, respectively; we do not use these labels to avoid confusion with the parameters introduced in (4.9). 


$$
\begin{aligned}
\mathcal{P}_{h^{+}}\left(B^{0} ; \tau\right)=\frac{\left|\mathcal{M}_{h}\right|^{2}}{2} e^{-\tau}\left\{\cos \omega \tau\left[-\frac{4 e^{-(A-D) \tau}}{\left|r_{S}+r_{L}\right|^{2}}-2 \mathcal{R} e\left(y_{h}\right)\right]\right. \\
+\sin \omega \tau\left[2 \mathcal{I} m\left(\mu_{h}\right)-\mathcal{R} e(B)+\frac{4 \delta}{\delta^{2}+\omega^{2}} \mathcal{I} m(C)\right] \\
+\cosh \delta \tau\left[\frac{4}{\left|r_{S}+r_{L}\right|^{2}}+2 \mathcal{R} e\left(y_{h}\right)\right] \\
\left.+\sinh \delta \tau\left[-2 \mathcal{R} e\left(\mu_{h}\right)+\frac{D}{\delta}-\frac{4 \omega}{\delta^{2}+\omega^{2}} \mathcal{I} m(C)\right]\right\},
\end{aligned}
$$

where the effects of the non-standard, dissipative phenomena are controlled by the dimensionless parameters $[c f .(3.22)]$

$$
A=\frac{\widetilde{A}}{\Gamma}, \quad B=\frac{\widetilde{B}}{\Delta m}, \quad C=\frac{\widetilde{C}}{\Gamma}, \quad D=\frac{\widetilde{D}}{\Gamma} .
$$

The expressions for $\mathcal{P}_{h^{-}}\left(\overline{B^{0}} ; \tau\right)$ and $\mathcal{P}_{h^{+}}\left(\overline{B^{0}} ; \tau\right)$ are obtained from $(5.5 b)$ and $(5.5 a)$, respectively, by changing the sign of $y_{h}$ and $C$, and letting $r_{S} \rightarrow 1 / r_{S}, r_{L} \rightarrow 1 / r_{L}$ and $\lambda_{h} \leftrightarrow \mu_{h}$. One can check that the formulas (5.5) reduce to those presented in [25] when adopting the particular phase convention for which

$$
r_{S} \simeq 1+2 \epsilon_{S}, \quad r_{L} \simeq 1+2 \epsilon_{L},
$$

and the quantities $\epsilon_{S}$ and $\epsilon_{L}$, parametrizing $C P T$ and $C P$ violation in "mixing", are considered small. Instead, we stress that the expressions in (5.5) are completely general, and manifestly independent of any phase-choice.

The probabilities $\mathcal{P}_{h}$ are directly accessible and can be studied in experiments performed at colliders. A preliminary investigation, assuming no dissipation, has already been performed at LEP in the framework of the approximation (5.7) and an upper bound on the magnitude of $\mathcal{R} e\left(\epsilon_{S}+\epsilon_{L}\right)$ has been obtained.[47, 48] Much more accurate studies can be performed both at the Tevatron and LHC, where the $\tau$-dependence in (5.5) can actually be observed; extrapolating from preliminary simulation estimates, sensitivities of a few percent on at least some of the parameters in (5.6) can be reasonably expected. $[49,50]$

Instead of analyzing directly the decay rates in (5.5), it might be more convenient to study specific asymmetries, constructed by taking suitable combinations of the $\mathcal{P}_{h}$ 's. Two independent simple asymmetries that can be formed with the probabilities $\mathcal{P}_{h}$ and are likely to be studied at colliders are:

$$
\begin{aligned}
& A_{C P T}(\tau)=\frac{\mathcal{P}_{h^{+}}\left(\overline{B^{0}} ; \tau\right)-\mathcal{P}_{h^{-}}\left(B^{0} ; \tau\right)}{\mathcal{P}_{h^{+}}\left(\overline{B^{0}} ; \tau\right)+\mathcal{P}_{h^{-}}\left(B^{0} ; \tau\right)} \\
& A_{T}(\tau)=\frac{\mathcal{P}_{h^{-}}\left(\overline{B^{0}} ; \tau\right)-\mathcal{P}_{h^{+}}\left(B^{0} ; \tau\right)}{\mathcal{P}_{h^{-}}\left(\overline{B^{0}} ; \tau\right)+\mathcal{P}_{h^{+}}\left(B^{0} ; \tau\right)}
\end{aligned}
$$


The first one is sensible to $C P T$ violating effects, by comparing the rate difference between the process $B^{0} \rightarrow B^{0}$ and its $C P T$-conjugate $\overline{B^{0}} \rightarrow \overline{B^{0}}$, while the second asymmetry signals the violation of time reversal by measuring the rate difference between the process $B^{0} \rightarrow \overline{B^{0}}$ and its time-conjugate $\overline{B^{0}} \rightarrow B^{0}$.

Unfortunately, these simple observables are not very sensitive to the presence of the non-standard, dissipative effects. For instance, by using the expressions in (5.5), one finds:

$$
\begin{aligned}
A_{C P T}(\tau)= & \frac{\left(\left|r_{S}\right|^{2}-\left|r_{L}\right|^{2}\right) \sinh \delta \tau+2 \mathcal{I} m\left(r_{S} r_{L}^{*}\right) \sin \omega \tau}{\left(\left|r_{S}\right|^{2}+\left|r_{L}\right|^{2}\right) \cosh \delta \tau+2 \mathcal{R} e\left(r_{S} r_{L}^{*}\right) \cos \omega \tau}+2 \mathcal{R} e\left(y_{h}\right) \\
& +\frac{1}{\cosh \delta \tau+\cos \omega \tau}\left[\mathcal{I} m\left(\lambda_{h}-\mu_{h}\right) \sin \omega \tau+\mathcal{R} e\left(\lambda_{h}-\mu_{h}\right) \sinh \delta \tau\right] \\
& -\left(\frac{\cosh \delta \tau-\cos \omega \tau}{\cosh \delta \tau+\cos \omega \tau}\right) \frac{4 \delta}{\delta^{2}+\omega^{2}} \mathcal{R} e(C)
\end{aligned}
$$

which depends on the dissipative parameters through $\mathcal{R} e(C)$. However, in (5.9) this parameter appears multiplied by a factor $\delta$, so that its contribution to $A_{C P T}(\tau)$ turns out to be suppressed with respect to the other terms; in particular, the approximation $\delta \approx 0$ would completely eliminate the presence of this parameter from (5.9). The same conclusion holds for the other asymmetry $A_{T}$. Therefore, observables of type (5.8) are not suitable for probing the presence of dissipative effects in neutral $B$-meson physics.

More complicated combinations of the probability rates $\mathcal{P}_{h}$ are needed in order to isolate the dissipative contributions. A particularly illuminating example is given by:

$$
A_{\Delta m}(\tau)=\frac{\left[\mathcal{P}_{h^{-}}\left(B^{0} ; \tau\right)-\mathcal{P}_{h^{-}}\left(\overline{B^{0}} ; \tau\right)\right]-\left[\mathcal{P}_{h^{+}}\left(B^{0} ; \tau\right)-\mathcal{P}_{h^{+}}\left(\overline{B^{0}} ; \tau\right)\right]}{\mathcal{P}_{h^{-}}\left(B^{0} ; \tau\right)+\mathcal{P}_{h^{-}}\left(\overline{B^{0}} ; \tau\right)+\mathcal{P}_{h^{+}}\left(B^{0} ; \tau\right)+\mathcal{P}_{h^{+}}\left(\overline{B^{0}} ; \tau\right)}
$$

Even in the approximation $\delta=0$, this asymmetry retains a distinctive dependence on the parameters that signal the presence of dissipation:

$$
A_{\Delta m}(\tau)=[\mathcal{R} e(\theta)]^{2}(1-\cos \omega \tau)+e^{-A \tau} \cos \omega \tau+\left[\mathcal{R} e(B)-\mathcal{I} m\left(\lambda_{h}+\mu_{h}\right)\right] \sin \omega \tau
$$

Assuming the validity of the $\Delta B=\Delta Q$ rule, and neglecting the quadratic dependence on the $C P T$-violating parameter $\theta$, the different time behaviours in $A_{\Delta m}(\tau)$ should allow a determination of the non-standard parameters $A$ and the real part of $B$. The actual accuracy of such a determination highly depends on the sensitivity of the measure of $A_{\Delta m}$. Although specific studies have not yet been performed for the form (5.11) of this asymmetry, from the results of available simulations one can nevertheless expect an accuracy in the determination of the various terms in $A_{\Delta m}$ of about a few percent.[49, 50] This sensitivity is already enough to give interesting bounds on $A$ and $\mathcal{R} e(B)$.

Nevertheless, the most simple and accurate tests on the extended dynamics in (3.1) that can be performed at colliders, using semileptonic decays, is based on the analysis of time-integrated rates, defined in general as

$$
\mathcal{P}_{f}(B)=\frac{1}{\Gamma} \int_{0}^{\infty} d \tau \mathcal{P}_{f}(B ; \tau)
$$


Despite most asymmetries constructed with these integrated probabilities suffer the same $\delta$-suppression problem mentioned before in discussing the observables in (5.8), a combination analogous to the one in (5.10) is again very sensible to the presence of dissipative parameters. An explicit evaluation gives, for $\delta=0$ :

$$
\begin{aligned}
A_{\Delta m}^{\prime}=\frac{1}{1+\omega^{2}}\left\{1+\omega^{2}[\mathcal{R} e(\theta)]^{2}\right. & +\omega\left[\mathcal{R} e(B)-\mathcal{I} m\left(\lambda_{h}+\mu_{h}\right)\right] \\
& \left.+\frac{1}{1+\omega^{2}}\left[\left(\omega^{2}-1\right) A-2 \omega^{2} D\right]\right\} .
\end{aligned}
$$

Assuming again the $\Delta B=\Delta Q$ rule and neglecting quadratic $C P T$-violating effects in "mixing" $\left([\mathcal{R} e(\theta)]^{2}=0\right)$, a measure of $A_{\Delta m}^{\prime}\left(1+\omega^{2}\right)$ not compatible with 1 would clearly signal the presence of non-standard effects. Such a test can be easily performed at colliders with high accuracy, in particular using the next generation dedicated $B$-meson experiments.

Further useful information on the dissipative parameters in (5.6) can be obtained by studying observables and asymmetries involving $B$-meson decays into hadronic final states $f$. The explicit form of such observables are in general more involved than the ones containing semileptonic decays, since now one can not resort to simplifying approximations as in (4.10). Nevertheless, when the final states $f$ exhibit a definite $C P$-parity $\zeta_{f}$, at least within a certain approximation, manageable expressions for the relevant asymmetries can be obtained.

The typical observable that can be constructed in this case involves the difference of the two probabilities in (5.2):

$$
A_{f}(\tau)=\frac{\mathcal{P}_{f}\left(B^{0} ; \tau\right)-\mathcal{P}_{f}\left(\overline{B^{0}} ; \tau\right)}{\mathcal{P}_{f}\left(B^{0} ; \tau\right)+\mathcal{P}_{f}\left(\overline{B^{0}} ; \tau\right)},
$$

where, for instance, $f$ can represent $D^{+} D^{-}, \pi^{+} \pi^{-}$or $J / \psi K$ final states. These asymmetries will be the target of very intensive experimental studies: in fact, they offer the possibility of clean tests of the standard model paradigm for $C P$-violation. On top of this, they turn out to be very sensible to the determination of the dissipative parameter $C$ in (5.6).

For simplicity, in presenting the explicit expression for the asymmetry $A_{f}(\tau)$ we shall take $\delta=0$ and retain only first order terms in the $C P T$-violating parameter $\mathcal{R} e(\theta)$. Within this approximation the two quantities $\lambda_{S}^{f}$ and $\lambda_{L}^{f}$ parametrizing the $B^{0}-\overline{B^{0}}$ decay into $f$ can be expressed in terms of a unique amplitude ratio,

$$
\lambda_{f}=\sqrt{\sigma^{*}} \frac{\mathcal{A}\left(\overline{B^{0}} \rightarrow f\right)}{\mathcal{A}\left(B^{0} \rightarrow f\right)},
$$

as follows

$$
\lambda_{S}^{f}=\lambda_{f}[1-\mathcal{R} e(\theta)], \quad \lambda_{L}^{f}=\lambda_{f}[1+\mathcal{R} e(\theta)] .
$$

Using these results in the computation of the probabilities in (5.2), one finally obtains:

$$
\begin{aligned}
A_{f}(\tau)=\frac{2 \mathcal{R} e\left(\lambda_{f}\right)}{1+\left|\lambda_{f}\right|^{2}} \mathcal{R} e(\theta) & +\frac{2 \zeta_{f}}{\omega} \mathcal{I} m(C)-\left[\frac{2 \mathcal{I} m\left(\lambda_{f}\right)}{1+\left|\lambda_{f}\right|^{2}}+\frac{2 \zeta_{f}}{\omega} \mathcal{R} e(C)\right] \sin \omega \tau \\
& +\left[\frac{1-\left|\lambda_{f}\right|^{2}}{1+\left|\lambda_{f}\right|^{2}}-\frac{2 \mathcal{R} e\left(\lambda_{f}\right)}{1+\left|\lambda_{f}\right|^{2}} \mathcal{R} e(\theta)-\frac{2 \zeta_{f}}{\omega} \mathcal{I} m(C)\right] \cos \omega \tau .
\end{aligned}
$$


The presence of dissipation manifests itself in the modification of the coefficients of the oscillating terms and in the presence of a $\tau$-independent piece. Neglecting $C P T$-violations in "mixing" $(\theta=0)$ and assuming a vanishingly small "direct" $C P$-violation, i.e. $\left|\lambda_{f}\right| \approx 1$, the different $\tau$-dependence in the three terms of $A_{f}(\tau)$ allows a determination of both $\mathcal{R} e(C)$ and $\mathcal{I} m(C)$, together with the $C P$-violating parameter $\mathcal{I} m\left(\lambda_{f}\right)$. Dedicated $B$ experiments at colliders should be able to identify the various $\tau$-dependence and therefore provide very stringent bounds on the parameter $C$.

On the other hand, integrated asymmetries, constructed from the probabilities in (5.12), are not very useful in probing the presence of dissipative effects in neutral $B$-meson decays. For instance, using the same approximations that lead to (5.17), the integrated asymmetry $A_{f}^{\prime}$ defined as in (5.14) takes the form:

$$
\begin{aligned}
A_{f}^{\prime}=\frac{2 \mathcal{R} e\left(\lambda_{f}\right)}{1+\left|\lambda_{f}\right|^{2}} \mathcal{R} e(\theta)+\frac{2 \zeta_{f}}{\omega} \mathcal{I} m(C) & -\frac{1}{1+\omega^{2}}\left\{\omega\left[\frac{2 \mathcal{I} m\left(\lambda_{f}\right)}{1+\left|\lambda_{f}\right|^{2}}+\frac{2 \zeta_{f}}{\omega} \mathcal{R} e(C)\right]\right. \\
& \left.-\frac{1-\left|\lambda_{f}\right|^{2}}{1+\left|\lambda_{f}\right|^{2}}+\frac{2 \mathcal{R} e\left(\lambda_{f}\right)}{1+\left|\lambda_{f}\right|^{2}} \mathcal{R} e(\theta)+\frac{2 \zeta_{f}}{\omega} \mathcal{I} m(C)\right\} .
\end{aligned}
$$

Even for vanishing $\mathcal{R} e(\theta)$ and $\left|\lambda_{f}\right|=1$, the measure of this asymmetry alone gives little information on the magnitude of $C$.

\section{CORRELATED MESON DECAYS}

The non-standard, dissipative effects described by the generalized dynamics (3.1) can be further analyzed in experiments involving correlated $B^{0}-\overline{B^{0}}$ mesons, at the so-called $B$-factories. Indeed, as we shall see, these set-ups appear particularly suitable for studying phenomena involving loss of quantum coherence.

In those experiments, correlated $B^{0}-\overline{B^{0}}$ mesons are produced from the decay of the $\Upsilon(4 S)$ resonance. Since the $\Upsilon$-meson has spin 1 , its decay into two spinless bosons produces an antisymmetric spatial state. In the $\Upsilon$-rest frame, the two neutral $B$-mesons are produced flying apart with opposite momenta; in the basis $\left|B^{0}\right\rangle,\left|\overline{B^{0}}\right\rangle$, the resulting state can be described by:

$$
\left|\psi_{A}\right\rangle=\frac{1}{\sqrt{2}}\left(\left|B^{0},-p\right\rangle \otimes\left|\overline{B^{0}}, p\right\rangle-\left|\overline{B^{0}},-p\right\rangle \otimes\left|B^{0}, p\right\rangle\right)
$$

The corresponding density operator $\rho_{A}$ can be abstractly expressed in terms of a projector:

$$
\rho_{A}=\left|\psi_{A}\right\rangle\left\langle\psi_{A}\right|
$$

As in the previous sections, we find it more practical to use the transformation introduced in $(3.12),(3.13)$ and pass to a different representation:

$$
\rho_{A} \rightarrow \widetilde{\rho}_{A}=\left[\widetilde{V}^{-1} \otimes \widetilde{V}^{-1}\right] \rho_{A}\left[\widetilde{V}^{\dagger-1} \otimes \widetilde{V}^{\dagger-1}\right]
$$


The $4 \times 4$ matrix $\widetilde{\rho}_{A}$ can then be explicitly expressed as:

$$
\widetilde{\rho}_{A}=\frac{1}{2\left|r_{S}+r_{L}\right|^{2}}\left[\widetilde{P}_{1} \otimes \widetilde{P}_{2}+\widetilde{P}_{2} \otimes \widetilde{P}_{1}-\widetilde{P}_{3} \otimes \widetilde{P}_{4}-\widetilde{P}_{4} \otimes \widetilde{P}_{3}\right]
$$

where

$$
\begin{array}{ll}
\widetilde{P}_{1}=\left(\begin{array}{ll}
1 & 0 \\
0 & 0
\end{array}\right), & \widetilde{P}_{2}=\left(\begin{array}{ll}
0 & 0 \\
0 & 1
\end{array}\right), \\
\widetilde{P}_{3}=\left(\begin{array}{ll}
0 & 1 \\
0 & 0
\end{array}\right), & \widetilde{P}_{4}=\left(\begin{array}{ll}
0 & 0 \\
1 & 0
\end{array}\right) .
\end{array}
$$

The evolution in time of the density matrix $\widetilde{\rho}_{A}$ can be analyzed using the single $B$ meson dynamics $\tilde{\rho}(t)$ discussed in Sect.3. We shall assume that once produced in a $\Upsilon$-decay, the two mesons evolve in time each according to the completely positive map $\gamma_{t}$ generated by (3.16)..$^{\dagger}$ This guarantees that the resulting dynamics is completely positive and of semigroup type. As already mentioned, this assures the positivity of the eigenvalues of any physical states at all times: this is an essential condition for the consistent description of any physical system.[23, 42]

The density matrix that describes a situation in which the first $B$-meson has evolved up to proper time $t_{1}$ and the second up to proper time $t_{2}$ is then given by:

$$
\begin{aligned}
\widetilde{\rho}_{A}\left(t_{1}, t_{2}\right) \equiv & \left(\gamma_{t_{1}} \otimes \gamma_{t_{2}}\right)\left[\widetilde{\rho}_{A}\right] \\
= & \frac{1}{2\left|r_{S}+r_{L}\right|^{2}}\left[\widetilde{P}_{1}\left(t_{1}\right) \otimes \widetilde{P}_{2}\left(t_{2}\right)+\widetilde{P}_{2}\left(t_{1}\right) \otimes \widetilde{P}_{1}\left(t_{2}\right)\right. \\
& \left.\quad-\widetilde{P}_{3}\left(t_{1}\right) \otimes \widetilde{P}_{4}\left(t_{2}\right)-\widetilde{P}_{4}\left(t_{1}\right) \otimes \widetilde{P}_{3}\left(t_{2}\right)\right],
\end{aligned}
$$

where $\widetilde{P}_{i}\left(t_{1}\right)$ and $\widetilde{P}_{i}\left(t_{2}\right), i=1,2,3,4$, represent the evolution according to (3.16) of the initial operators $\widetilde{P}_{i}$ in (6.5), up to the time $t_{1}$ and $t_{2}$, respectively (see Appendix C).

The two $B$-mesons that come from a decay of an $\Upsilon$ are quantum-mechanically entangled, in a way very similar to that of two spin $1 / 2$ particles coming from a singlet state. As in that case, correlated measures on the two particles become physically significant. Indeed, the typical observables that can be studied at $B$-factories are double decay rates, i.e. the probabilities $\mathcal{G}\left(f_{1}, t_{1} ; f_{2}, t_{2}\right)$ that a meson decays into a final state $f_{1}$ at proper time $t_{1}$, while the other meson decays into the final state $f_{2}$ at proper time $t_{2}$. They can be computed using:

$$
\mathcal{G}\left(f_{1}, t_{1} ; f_{2}, t_{2}\right)=\operatorname{Tr}\left[\left(\widetilde{\mathcal{O}}_{f_{1}} \otimes \widetilde{\mathcal{O}}_{f_{2}}\right) \widetilde{\rho}_{A}\left(t_{1}, t_{2}\right)\right]
$$

where $\widetilde{\mathcal{O}}_{f_{1}}, \widetilde{\mathcal{O}}_{f_{2}}$ represent $2 \times 2$ hermitian matrices describing the decay of a single meson into the final states $f_{1}, f_{2}$, respectively. They can be identified with one of the two matrices

$\dagger$ Although other possibilities are in principle conceivable, this choice is the most natural one: it assures a consistent single meson dynamics when tracing over the degrees of freedom of one of the two $B$-mesons.[23] 
in (4.6) and (4.8). Let us denote with $\widetilde{\mathcal{O}}_{f}^{i}, i=1,2,3,4$, the entries of the matrix $\widetilde{\mathcal{O}}_{f}$ when projected along the operators $\widetilde{P}_{i}$ :

$$
\widetilde{\mathcal{O}}_{f}=\sum_{i=1}^{4} \widetilde{\mathcal{O}}_{f}^{i} \widetilde{P}_{i}
$$

The observables (6.7) can then be rewritten as

$$
\mathcal{G}\left(f_{1}, t_{1} ; f_{2}, t_{2}\right)=\frac{1}{2\left|r_{S}+r_{L}\right|^{2}} \sum_{i, j=1}^{4} \widetilde{\mathcal{O}}_{f_{1}}^{i} \widetilde{\mathcal{O}}_{f_{2}}^{j} \mathcal{P}_{i j}\left(t_{1}, t_{2}\right)
$$

where the elementary probabilities

$$
\begin{aligned}
\mathcal{P}_{i j}\left(t_{1}, t_{2}\right)=\operatorname{Tr}\left\{\widetilde{P}_{i} \widetilde{P}_{1}\left(t_{1}\right)\right\} \operatorname{Tr}\left\{\widetilde{P}_{j} \widetilde{P}_{2}\left(t_{2}\right)\right\}+\operatorname{Tr}\left\{\widetilde{P}_{i} \widetilde{P}_{2}\left(t_{1}\right)\right\} \operatorname{Tr}\left\{\widetilde{P}_{j} \widetilde{P}_{1}\left(t_{2}\right)\right\} \\
-\operatorname{Tr}\left\{\widetilde{P}_{i} \widetilde{P}_{3}\left(t_{1}\right)\right\} \operatorname{Tr}\left\{\widetilde{P}_{j} \widetilde{P}_{4}\left(t_{2}\right)\right\}-\operatorname{Tr}\left\{\widetilde{P}_{i} \widetilde{P}_{4}\left(t_{1}\right)\right\} \operatorname{Tr}\left\{\widetilde{P}_{j} \widetilde{P}_{3}\left(t_{2}\right)\right\},
\end{aligned}
$$

can be easily computed to any given accuracy using the solution of the evolution equation (3.16) collected in Appendix B.

The expressions for the double decay rates in (6.9) can be compared with the results of the experiment. However, much of the analysis at $B$-factories is carried out using integrated distributions at fixed time interval $t=t_{1}-t_{2}$; this is a consequence of the short $B$-mesons lifetime and rapid $B^{0}-\overline{B^{0}}$ oscillations, that does not allow a precise enough study of the double time dependence in (6.9). One is then forced to construct single-time distributions, defined by

$$
\begin{aligned}
\Gamma\left(f_{1}, f_{2} ; t\right) & \equiv \int_{0}^{\infty} d t^{\prime} \mathcal{G}\left(f_{1}, t^{\prime}+t ; f_{2}, t^{\prime}\right) \\
& =\frac{1}{2\left|r_{S}+r_{L}\right|^{2}} \sum_{i, j=1}^{4} \widetilde{\mathcal{O}}_{f_{1}}^{i} \widetilde{\mathcal{O}}_{f_{2}}^{j} \Pi_{i j}(t)
\end{aligned}
$$

where

$$
\Pi_{i j}(t)=\int_{0}^{\infty} d t^{\prime} \mathcal{P}_{i j}\left(t^{\prime}+t, t^{\prime}\right)
$$

and $t$ is taken to be positive. For negative $t$, one defines:

$$
\Gamma\left(f_{1}, f_{2} ;-|t|\right)=\int_{0}^{\infty} d t^{\prime} \mathcal{G}\left(f_{1}, t^{\prime}-|t| ; f_{2}, t^{\prime}\right) \theta\left(t^{\prime}-|t|\right)
$$

the presence of the step-function is necessary since the evolution is of semigroup type, with forward in time propagation, starting from zero (we can not propagate a $B$-meson before it is created in a $\Upsilon$-decay). In this case, one easily finds:

$$
\Gamma\left(f_{1}, f_{2} ;-|t|\right)=\Gamma\left(f_{2}, f_{1} ;|t|\right) .
$$


In the following, we shall always assume: $t \geq 0$.

The explicit form of the single-time probabilities $\Pi_{i j}(t)$ in $(6.12)$ can be found in Appendix C; inserting these in (6.11), one is then able to compute the evolution in $t$ of the double decay rate $\Gamma\left(f_{1}, f_{2} ; t\right)$ for any specific decay state $f_{1}$ and $f_{2}$. Before presenting the results for some experimentally relevant cases, let us note that in general the non-standard dynamics in (3.16) gives results for $\Gamma\left(f_{1}, f_{2} ; t\right)$ that are quite different from those obtained in the usual case $($ i.e. in absence of $\widetilde{\mathcal{L}})$. The most striking difference arises when the final states coincide $f_{1}=f_{2}=f$ and $t$ approaches zero. Due to the antisymmetry of the initial state $\left|\psi_{A}\right\rangle$ in $(6.1)$, quantum mechanics predicts a vanishing value for $\Gamma(f, f ; 0)$, while in general this is not the case for the completely positive dynamics generated by (3.1). This explains why correlated mesons systems turn out to be the most natural place to look for effects leading to loss of phase coherence and dissipation.

The quantities $\Gamma(f, f ; t)$, with $t$ small, are therefore very sensitive to the dissipative parameters in (3.22). This can be explicitly shown by considering the following combination of decay rates involving semileptonic final states:

$$
\mathcal{R}(\tau)=\frac{\Gamma\left(h^{+}, h^{+} ; \tau\right)+\Gamma\left(h^{-}, h^{-} ; \tau\right)}{\Gamma\left(h^{+}, h^{-} ; \tau\right)+\Gamma\left(h^{-}, h^{+} ; \tau\right)},
$$

where again the variable $\tau=t \Gamma$ has been introduced. Neglecting terms containing $\delta$ times small parameters, one finds:

$$
\begin{aligned}
\mathcal{R}(\tau)= & \frac{\left(1+\left|r_{S}\right|^{2}\left|r_{L}\right|^{2}\right)(\cosh \delta \tau-\cos \omega \tau)}{\left(\left|r_{S}\right|^{2}+\left|r_{L}\right|^{2}\right) \cosh \delta \tau+2 \mathcal{R} e\left(r_{S} r_{L}^{*}\right) \cos \omega \tau} \\
& +\frac{2}{(\cos \omega \tau+1)^{2}}\left[A(1+\tau) \cos \omega \tau-(\omega \cos \omega \tau+\sin \omega \tau) \mathcal{R} e\left(\frac{B}{1-i \omega}\right)\right] .
\end{aligned}
$$

As $\tau$ approaches zero, the first term becomes vanishingly small, while the surviving pieces are all proportional to the dissipative parameters, so that:

$$
\mathcal{R}(0)=\frac{1}{2}\left\{A+\frac{\omega}{1+\omega^{2}}[\omega \mathcal{I} m(B)-\mathcal{R} e(B)]\right\} \text {. }
$$

A non vanishing result in the measure of this quantity would clearly signal the presence of dissipation. However, to obtain more detailed information on the magnitude of the non-standard parameters, one has to study the full $\tau$-dependence in (6.16), and further analyze the behaviour of other observables that can be constructed out of the double probabilities $\Gamma$.

For instance, one can reverse the sign in the numerator of (6.15) and study the asymmetry:

$$
\mathcal{A}_{T}(\tau)=\frac{\Gamma\left(h^{+}, h^{+} ; \tau\right)-\Gamma\left(h^{-}, h^{-} ; \tau\right)}{\Gamma\left(h^{+}, h^{-} ; \tau\right)+\Gamma\left(h^{-}, h^{+} ; \tau\right)} .
$$

Unfortunately, the same phenomenon already noticed in the case of single meson asymmetries occurs also here: the dependence on the dissipative parameters is always suppressed 
by a factor $\delta$, so that it becomes extremely difficult to extract information about the non-standard effects from these observables.

The situation is different for the asymmetry $\mathcal{A}_{\Delta m}(\tau)$, analogous to the one in $(5.10)$ for single-meson systems:

$$
\mathcal{A}_{\Delta m}(\tau)=\frac{\left[\Gamma\left(h^{+}, h^{-} ; \tau\right)+\Gamma\left(h^{-}, h^{+} ; \tau\right)\right]-\left[\Gamma\left(h^{+}, h^{+} ; \tau\right)+\Gamma\left(h^{-}, h^{-} ; \tau\right)\right]}{\Gamma\left(h^{+}, h^{+} ; \tau\right)+\Gamma\left(h^{-}, h^{-} ; \tau\right)+\Gamma\left(h^{+}, h^{-} ; \tau\right)+\Gamma\left(h^{-}, h^{+} ; \tau\right)} .
$$

In fact, even in the case $\delta=0$, this asymmetry has a very distinctive dependence on the non-standard parameters:

$$
\mathcal{A}_{\Delta m}(\tau)=\frac{e^{-A \tau}}{1+A} \cos \omega \tau+[\mathcal{R} e(\theta)]^{2}(1-\cos \omega \tau)+(\omega \cos \omega \tau+\sin \omega \tau) \mathcal{R} e\left(\frac{B}{1-i \omega}\right)
$$

Thanks to the different time behaviour, an experimental study of this observable should allow to extract precise information on $A$ and the combination $\omega \mathcal{I} m(B)-\mathcal{R} e(B)$; the analysis of $\mathcal{R}(\tau)$ in (6.16) should then provide an estimate on the parameter $D$.

The asymmetry in (6.19) is presently under intensive study at $B$-factories:[51-53] it is used to obtain a precise determination of the mass difference $\Delta m$, since in absence of dissipation: $\mathcal{A}_{\Delta m}(\tau)=\cos \omega \tau$. Although an accurate analysis is certainly needed in order to estimate precisely the sensitivity of those experiments to the different $\tau$-dependent functions in (6.20), on the basis of estimated data acquisition and relative errors, one can reasonably expect that the actual measured data will at the end constrain the dissipative constants $A$ and $\omega \mathcal{I} m(B)-\mathcal{R} e(B)$ to a few percent level. This would allow to establish significant limits on the presence of dissipative effects in the $B^{0}-\overline{B^{0}}$ system.

To get information on the magnitude of the fourth parameter $C$ in (5.6), one has to study observables that involve also hadronic decay channels, in particular those with definite $C P$-parity. A typical asymmetry that is measured at $B$-factories involves double decay rates $\Gamma\left(f_{1}, f_{2} ; \tau\right)$ in which one of the final states is semileptonic and the other is hadronic:

$$
\mathcal{A}_{f}(\tau)=\frac{\Gamma\left(h^{+}, f ; \tau\right)-\Gamma\left(h^{-}, f ; \tau\right)}{\Gamma\left(h^{+}, f ; \tau\right)+\Gamma\left(h^{-}, f ; \tau\right)} .
$$

Since the two mesons are quantum mechanically correlated, neglecting $\Delta B=\Delta Q$ violating effects, the semileptonic decay effectively "tags" the flavour of the meson that decays into $f$. In view of this, one usually consider the asymmetry in (6.21) and the one in (5.14) for single meson decays as the same observable; [1, 2] for instance, when $f=J / \psi K_{S}$ both asymmetries can be directly expressible in terms of one of the angles parametrizing $C P$ violation in the standard model. The situation radically changes in presence of dissipation; as already stressed, the form of the double decay rates $\Gamma\left(f_{1}, f_{2} ; \tau\right)$ turns out to be very different from that predicted by ordinary quantum mechanics and this makes the asymmetry (6.21) distinct from that in (5.14).

In presenting the explicit expression of $\mathcal{A}_{f}(\tau)$, one can set $\delta=0$, since this choice does not affect the dependence on the dissipative parameters. Further, we shall assume that $C P T$-violation in "mixing" be small, and therefore neglect terms containing powers of 
the parameter $\mathcal{R} e(\theta)$ higher then one. Within these approximations, one explicitly finds:

$$
\begin{aligned}
\mathcal{A}_{f}(\tau)=\frac{2 \mathcal{R} e\left(\lambda_{f}\right)}{1+\left|\lambda_{f}\right|^{2}} & \mathcal{R} e(\theta)+\zeta_{f} \mathcal{R} e\left(\lambda_{h}-\mu_{h}+2 \zeta_{f} y_{h}\right)-\frac{2 \zeta_{f}}{\omega} \mathcal{I} m(C) \\
+ & {\left[\frac{2 \mathcal{I} m\left(\lambda_{f}\right)}{1+\left|\lambda_{f}\right|^{2}}-\frac{2 \zeta_{f}}{\omega} \mathcal{I} m\left(\frac{2 i+\omega}{2+i \omega} C\right)\right] \sin \omega \tau } \\
+ & {\left[\frac{1-\left|\lambda_{f}\right|^{2}}{1+\left|\lambda_{f}\right|^{2}}-\frac{2 \mathcal{R} e\left(\lambda_{f}\right)}{1+\left|\lambda_{f}\right|^{2}} \mathcal{R} e(\theta)-\frac{2 \zeta_{f}}{\omega} \mathcal{R} e\left(\frac{2 i+\omega}{2+i \omega} C\right)\right] \cos \omega \tau }
\end{aligned}
$$

where the decay parameter $\lambda_{f}$ is defined in (5.15), while $\zeta_{f}$ represents again the intrinsic $C P$ parity of the state $f$.

Assuming the validity of the $\Delta B=\Delta Q$ rule and neglecting $C P T$-violating effects in "mixing" $(\theta=0)$ as well as "direct" $C P$-violations $\left(\left|\lambda_{f}\right|=1\right)$, a fit of $(6.22)$ with experimental data allows the determination of both $\mathcal{R} e(C)$ and $\mathcal{I} m(C)$, together with $\mathcal{I} m\left(\lambda_{f}\right)$. The asymmetry $\mathcal{A}_{f}$, in particular for the final state $f=J / \psi K_{S}$, will be measured with increasing accuracy at $B$-factories, so that a high sensitivity on the different $\tau$ dependences in (6.22) is expected.[51] Therefore, the study of $\mathcal{A}_{f}$ could result in one of the best tests on the presence of dissipative effects in $B$ physics.

As remarked before, the form of $\mathcal{A}_{f}(\tau)$ in (6.22) is valid only for $\tau \geq 0$. For negative times, one has to use the an analogous expression, obtained from the definition in (6.21) by exchanging the positions of the semileptonic and $f$ final states in the probabilities $\Gamma$. Explicit computations gives:

$$
\begin{aligned}
\mathcal{A}_{f}(-|\tau|)=\frac{2 \mathcal{R} e\left(\lambda_{f}\right)}{1+\left|\lambda_{f}\right|^{2}} & \mathcal{R} e(\theta)+\zeta_{f} \mathcal{R} e\left(\lambda_{h}-\mu_{h}+2 \zeta_{f} y_{h}\right)-\frac{2 \zeta_{f}}{\omega} \mathcal{R} e\left(\frac{2 i+\omega}{2+i \omega} C\right) \\
- & {\left[\frac{2 \mathcal{I} m\left(\lambda_{f}\right)}{1+\left|\lambda_{f}\right|^{2}}+\frac{2 \zeta_{f}}{\omega} \mathcal{R} e(C)\right] \sin \omega|\tau| } \\
+ & {\left[\frac{1-\left|\lambda_{f}\right|^{2}}{1+\left|\lambda_{f}\right|^{2}}-\frac{2 \mathcal{R} e\left(\lambda_{f}\right)}{1+\left|\lambda_{f}\right|^{2}} \mathcal{R} e(\theta)-\frac{2 \zeta_{f}}{\omega} \mathcal{I} m(C)\right] \cos \omega|\tau| . }
\end{aligned}
$$

Note that the two expressions (6.22) and (6.23) coincide at $\tau=0$, while in absence of dissipation $(C=0),(6.23)$ can be obtained from (6.22) by letting $\tau \rightarrow-\tau$; the fact that this no longer true for $C \neq 0$ is a clear sign of presence of irreversibility.

The observables involving correlated $B$-mesons that have been discussed so far are accessible at the so-called asymmetric $B$-factories, where the $\Upsilon$-decays take place in a boosted reference frame with respect to the laboratory. This allows the subsequent decays of the two neutral $B$ mesons to be physically separated and therefore a good determination of the time-difference $\tau$. In symmetric $B$-factories, this measure is impossible: the two mesons decay too quickly to allow a complete reconstruction of the events. One has then to resort to time-independent probabilities, obtained by further integrating the rates $\Gamma\left(f_{1}, f_{2} ; t\right)$ in $(6.11)$ :

$$
\Gamma\left(f_{1}, f_{2}\right)=\int_{0}^{\infty} d t \Gamma\left(f_{1}, f_{2} ; t\right)
$$


The asymmetries that can be constructed out of these quantities are not very sensitive to the non-standard parameters, because of the suppression by factors $\delta$. Nevertheless, one can still form useful observables involving semileptonic final states; these are the total $B^{0}$ $\overline{B^{0}}$ mixing probability:

$$
\chi_{B}=\frac{\Gamma\left(h^{+}, h^{+}\right)+\Gamma\left(h^{-}, h^{-}\right)}{\Gamma\left(h^{+}, h^{+}\right)+\Gamma\left(h^{-}, h^{-}\right)+\Gamma\left(h^{+}, h^{-}\right)+\Gamma\left(h^{-}, h^{+}\right)},
$$

and the ratio of the total, same-sign to opposite-sign semileptonic rates:

$$
R_{B}=\frac{\Gamma\left(h^{+}, h^{+}\right)+\Gamma\left(h^{-}, h^{-}\right)}{\Gamma\left(h^{+}, h^{-}\right)+\Gamma\left(h^{-}, h^{+}\right)} .
$$

Both observables have a quadratic dependence on $\delta$; the approximation $\delta=0$ is therefore very accurate. With this choice, one explicitly finds:

$$
\begin{aligned}
& \chi_{B}=\frac{\omega^{2}}{2\left(1+\omega^{2}\right)}\left\{1-[\mathcal{R} e(\theta)]^{2}+\frac{2}{\omega^{2}\left(1+\omega^{2}\right)} X\right\} \\
& R_{B}=\frac{\omega^{2}\left(1-[\mathcal{R} e(\theta)]^{2}\right)}{2+\omega^{2}\left(1+[\mathcal{R} e(\theta)]^{2}\right)}+\frac{4}{\left(2+\omega^{2}\right)^{2}} X,
\end{aligned}
$$

where the dependence on the non-standard, dissipative parameters occurs via the combination:

$$
X=A+\omega^{2} D+\omega[\omega \mathcal{I} m(B)-\mathcal{R} e(B)] .
$$

Independent measures of these two quantities would provide a way to estimate both $[\mathcal{R} e(\theta)]^{2}$ and $X$, and therefore give limits on both dissipative and $C P T$-violating effects. Unfortunately, both observables are not very well known; $\chi_{B}$ is the better determined parameter and the most recent data ${ }^{\dagger}$ give: $\chi_{B}=0.198 \pm 0.019$. [54] Assuming $[\mathcal{R} e(\theta)]^{2}$ to be negligible and using the world average for $\omega,[55]$ from $(6.27 a)$ one gets the estimate: $X=(5.7 \pm 4.8) \times 10^{-2}$. $^{-}$The accuracy on the determination of $X$ will be greatly improved when also the measurements at the asymmetric $B$-factories will be available. Indeed, preliminary estimates of about one percent sensitivity to $R_{B}$ have been reported to be attainable in [38], while a conservative estimate of two percent accuracy in the measure of $\chi_{B}$ is indicated in [40]. If confirmed by the actual data, these sensitivities will allow a determination of the combination $X$ with about a few percent accuracy, providing an interesting test on the presence of non-standard effects in $B$-physics.

$\dagger$ The determination of $\chi_{B}$ in [54] actually involves so-called semileptonic-type decays [36] rather than pure semileptonic final states; one can check that the form $(6.27 a)$ for the observable $\chi_{B}$ is valid also in that case.

$\ddagger$ When $a=0$, the inequalities (2.13) further imply $\alpha=\gamma$ and $b=c=\beta=0$, or equivalently $A=B=D, C=0$. In this simplified case, an estimate on the variable $X$ translates into a corresponding one for the surviving dissipative parameter $\alpha$; in particular, from the above quoted value for $X$, one has: $\alpha=(4.5 \pm 3.8) \times 10^{-14} \mathrm{GeV}$. 


\section{DISCUSSION}

The description of open quantum systems in terms of quantum dynamical semigroups allows a general and consistent approach to the study of physical phenomena leading to irreversibility and dissipation. When applied to the analysis of the propagation and decay of neutral meson systems, it gives precise predictions on the behaviour of relevant physical observables: the new, dissipative phenomena manifest themselves through a set of phenomenological parameters, $a, b, c, \alpha, \beta$ and $\gamma$, whose presence can be experimentally probed.

Indeed, as discussed at length in the previous sections, various observables involving $B^{0}-\overline{B^{0}}$ decays can be identified as being particularly sensitive to the new, non-standard effects. These observables will be measured with great accuracy in the new generation of dedicated $B$-experiments, both at colliders (CDF-II, HERA-B, BTeV, LHC-b) and at $B$-factories (BaBar, Belle, CLEO-III), so that stringent bounds on the dissipative effects can be expected in the future.

From the experimental point of view, the actual visibility of these effects clearly depends on the magnitude of the parameters $a, b, c, \alpha, \beta$ and $\gamma$. A pure phenomenological approach can not provide such information. However, in the framework of open quantum systems, the neutral $B$-mesons are described as subsystems in interaction with an environment. In such instances, the effects of irreversibility and dissipation can be roughly estimated to be proportional to the square of the $B$-meson mass divided by the characteristic energy scale of the environment. Assimilating this scale to the Planck mass results in a very small estimate for the magnitude of the dissipative parameters, roughly of order $10^{-18} \mathrm{GeV}$. However, the sophistication of the experiments mentioned before is so high, that the sensitivity needed to provide useful constraints on such tiny effects should be reached in just a few years of data taking.[49-53]

Being based on the general theory of open systems, the above estimate on the magnitude of the non-standard, dissipative effects is rather robust and quite independent from the details of the actual dynamics that drives the interaction between subsystem and environment. Nevertheless, it has been recently questioned,[56] on the basis of the similarity of a simplified version of the evolution equation in (3.1) with those describing the dynamical reduction of the wave-packet. We point out that this analogy is only superficial: the physical effects leading to dissipation in open systems are clearly distinct from those advocated as responsible for the reduction process; and indeed, the quantum dynamical semigroup generated by (3.1) can not describe dynamical reduction phenomena.

The arguments of [56] have been further used in [57] to support the claim that nonlinear evolution equations should be used to analyze dissipative effects in neutral meson systems, although also there dynamics of the form (2.2) are nevertheless adopted at the end. In the framework of open systems, the situation can be easily clarified.

The dynamics of a small system $S$ in interaction with a large environment $E$ is in general very complex and can not be described in terms of evolution equations that are local in time: possible initial correlations and the continuous exchange of energy as well as entropy between the $S$ and $E$ produce memory effects and non-linear phenomena. Nevertheless, when the typical time scale in the evolution of the subsystem $S$ is much larger than the characteristic time correlations in the environment, the subdynamics simplifies and a 
mathematically precise description in terms of quantum dynamical semigroups naturally emerges.[4-6]

This description is very general and is applicable to all physical situations for which the interaction between $S$ and $E$ can be considered to be weak and for times for which non-linear disturbances due to possible initial correlations have disappeared.[7] These are precisely the conditions that are expected to be fulfilled in neutral meson systems: the characteristic time correlations in the environment, induced by the fundamental (e.g. gravitational or "stringy") dynamics, is certainly much smaller than the neutral meson lifetime, and the interaction between mesons and environment is for sure weak (its effects have not yet been detected). Furthermore, this open system paradigm automatically assures the fulfillment of basic physical properties, as forward in time composition and entropy increase (irreversibility). Therefore, the physical motivations for adopting a quantum dynamical semigroup description of the extended neutral-meson effective dynamics appear to be rather compelling and general.

As a final remark, let us point out that once effective dynamics generated by equations of the form (2.2) are accepted, the condition of complete positivity is then absolutely necessary for a physically consistent description of the neutral meson system. Indeed, time evolutions that do not satisfy this property unavoidably gives unphysical results.[42] This can be easily shown by examining the sign of the eigenvalues of of the density matrix $\widetilde{\rho}_{A}(t, t)$ representing a correlated meson state. In fact, one of the properties that any density matrix needs to satisfy is that its eigenvalues be non-negative, for all times; without this basic requirement, its standard probability interpretation would be meaningless.

As an example, following $[28,19,20]$, let us consider a dynamics for $\widetilde{\rho}_{A}$ generated by single-meson evolution equation of the form (3.1), where $\mathcal{L}$ is as in (2.14), but with vanishing $a, b$ and $c$. The conditions of complete positivity in (2.13) are then clearly violated (unless $\alpha=\gamma$ and $\beta=0$ ). For $\mathcal{H}=0$, the eigenvalues $\lambda_{i}(t), i=1,2,3$, 4, of the $4 \times 4$ matrix $\widetilde{\rho}_{A}(t, t)$ can be explicitly obtained; being physical quantities, they can be computed in any phase convention, and in particular in the one for which the dissipative parameters in (3.22) take the simplified form $\widetilde{A}=\widetilde{B}=\alpha, \widetilde{C}=i \beta$ and $\widetilde{D}=\gamma$. Then, one has:

$$
\begin{aligned}
& \lambda_{1,2}(t)=2 \pm\left\{\left[E_{+}(t)\right]^{2}+\left[E_{-}(t)\right]^{2}+2[F(t)]^{2}\right\} \\
& \lambda_{3,4}(t)= \pm\left[E_{+}(t)+E_{-}(t)\right]\left\{\left[E_{+}(t)-E_{-}(t)\right]^{2}+4[F(t)]^{2}\right\}^{1 / 2}
\end{aligned}
$$

where

$$
\begin{aligned}
& E_{ \pm}(t)=\frac{1}{\nu_{+}-\nu_{-}}\left[\left(\nu_{+}+2 \alpha\right) e^{\nu_{ \pm} t}-\left(\nu_{-}+2 \alpha\right) e^{\nu_{\mp} t}\right] \\
& F(t)=\frac{2 \beta}{\nu_{+}-\nu_{-}}\left[e^{\nu_{-} t}-e^{\nu_{+} t}\right]
\end{aligned}
$$

and $\nu_{ \pm}=-(\alpha+\gamma) \pm \sqrt{(\alpha-\gamma)^{2}+4 \beta^{2}}$ are both negative due to the simple positivity condition $\alpha \gamma \geq \beta^{2}$. As clear from the previous formulas, for $t \neq 0$ two of the eigenvalues of $\widetilde{\rho}_{A}(t, t)$ are always negative, signaling the presence of unphysical "negative probabilities". When complete positivity is enforced though, one has $\nu_{+}=\nu_{-}$and all eigenvalues in (7.1) remain non-negative for all times. 
These results offer a further motivation for studying generalized dynamics of the form (3.1) at meson factories. These set-ups are in fact high-performance quantum interferometers: at least in principle, they can clarify from the experimental point of view the role of the condition of complete positivity in the time evolution of correlated mesons. [42] As shown by the simple example above, this is not just a mere technical question: it is crucial to our physical understanding of the quantum dynamics of open systems.

\section{APPENDIX A}

As explained in the text, in order to find explicit solutions for the evolution equation (3.1), it is convenient to make a change of basis as described in (3.14). The resulting dynamical equation has a simplified hamiltonian part $\mathcal{H}_{0}$, while the dissipative piece becomes more involved: $\widetilde{\mathcal{L}}=\mathcal{V} \mathcal{L} \mathcal{V}^{-1}$. Its entries can be expressed as linear combinations of the dissipative parameters $a, b, c, \alpha, \beta$ and $\gamma$. One explicitly finds:

$$
\widetilde{\mathcal{L}}=\frac{1}{\left|r_{S}+r_{L}\right|^{2}}\left[\begin{array}{cccc}
\Lambda & \Sigma & \Delta & \Delta^{*} \\
\Xi & \Lambda & \Phi & \Phi^{*} \\
-\Phi^{*} & -\Delta^{*} & \Omega & \Theta \\
-\Phi & -\Delta & \Theta^{*} & \Omega
\end{array}\right]
$$

where

$$
\begin{aligned}
& \Lambda=a\left(\left|r_{S}\right|^{2}-1\right)\left(\left|r_{L}\right|^{2}-1\right)+2 \mathcal{R} e\left\{(c-i b)\left[\left(\left|r_{L}\right|^{2}-1\right) r_{S}-\left(\left|r_{S}\right|^{2}-1\right) r_{L}\right]\right\} \\
& -2(\alpha+\gamma) \mathcal{R} e\left(r_{S} r_{L}^{*}\right)+2 \mathcal{R} e\left\{(\alpha-\gamma+2 i \beta) r_{S} r_{L}\right\}, \\
& \Sigma=a\left(\left|r_{L}\right|^{2}-1\right)^{2}-4 \mathcal{R} e\left\{(c-i b)\left(\left|r_{L}\right|^{2}-1\right) r_{L}\right\}+2(\alpha+\gamma)\left|r_{L}\right|^{2} \\
& -2 \mathcal{R} e\left\{(\alpha-\gamma+2 i \beta) r_{L}^{2}\right\} \\
& \Xi=a\left(\left|r_{S}\right|^{2}-1\right)^{2}+4 \mathcal{R} e\left\{(c-i b)\left(\left|r_{S}\right|^{2}-1\right) r_{S}\right\}+2(\alpha+\gamma)\left|r_{S}\right|^{2} \\
& -2 \mathcal{R} e\left\{(\alpha-\gamma+2 i \beta) r_{S}^{2}\right\} \\
& \Delta=a\left(\left|r_{L}\right|^{2}-1\right)\left(r_{S} r_{L}^{*}+1\right)+(\alpha+\gamma)\left(r_{S}-r_{L}\right) r_{L}^{*}+(c-i b)\left(r_{S}-r_{L}-2 r_{S}\left|r_{L}\right|^{2}\right) \\
& +(c+i b)\left[\left(r_{L}-r_{S}\right) r_{L}^{*}-2\right] r_{L}^{*}+(\alpha-\gamma-2 i \beta) r_{L}^{* 2}-(\alpha-\gamma+2 i \beta) r_{S} r_{L},
\end{aligned}
$$




$$
\begin{gathered}
\Phi=a\left(\left|r_{S}\right|^{2}-1\right)\left(r_{S} r_{L}^{*}+1\right)+(\alpha+\gamma)\left(r_{L}^{*}-r_{S}^{*}\right) r_{S}+(c-i b)\left[\left(r_{L}^{*}-r_{S}^{*}\right) r_{S}+2\right] r_{S} \\
+(c+i b)\left(r_{S}^{*}-r_{L}^{*}+2\left|r_{S}\right|^{2} r_{L}^{*}\right)-(\alpha-\gamma-2 i \beta) r_{S}^{*} r_{L}^{*}+(\alpha-\gamma+2 i \beta) r_{S}^{2}, \\
\begin{array}{r}
\Theta=-a\left(r_{S}^{*} r_{L}+1\right)^{2}-2(c-i b)\left(r_{S}^{*} r_{L}+1\right) r_{L}+2(c+i b)\left(r_{S}^{*} r_{L}+1\right) r_{S}^{*}+2(\alpha+\gamma) r_{S}^{*} r_{L} \\
+(\alpha-\gamma-2 i \beta) r_{S}^{*}+(\alpha-\gamma+2 i \beta) r_{L}^{2}
\end{array} \\
\begin{array}{r}
\Omega=-a\left|r_{S} r_{L}^{*}+1\right|^{2}+2 \mathcal{R} e\left\{(c-i b)\left[\left(\left|r_{S}\right|^{2}-1\right) r_{L}-\left(\left|r_{L}\right|^{2}-1\right) r_{S}\right]\right\} \\
-(\alpha+\gamma)\left(\left|r_{S}\right|^{2}+\left|r_{L}\right|^{2}\right)-2 \mathcal{R} e\left\{(\alpha-\gamma+2 i \beta) r_{S} r_{L}\right\} .
\end{array}
\end{gathered}
$$

Recalling the discussion in Section 3, an independent phase change of the basis vectors, $\left|B^{0}\right\rangle \rightarrow e^{i \phi}\left|B^{0}\right\rangle,\left|\overline{B^{0}}\right\rangle \rightarrow e^{i \bar{\phi}}\left|\overline{B^{0}}\right\rangle$, induces a transformation on the entries of the matrix $\mathcal{L}$ in (2.14), realized by the operator $\mathcal{U}_{\phi}$ in (3.6); explicitly, one finds:

$$
(c-i b) \rightarrow e^{-i(\phi-\bar{\phi})}(c-i b), \quad(\alpha-\gamma-2 i \beta) \rightarrow e^{2 i(\phi-\bar{\phi})}(\alpha-\gamma-2 i \beta),
$$

while $a$ and $\alpha+\gamma$ remain unchanged. Similarly, also the two ratios $r_{S}$ and $r_{L}$ in (2.9) change, according to the rule:

$$
r_{S} \rightarrow e^{i(\phi-\bar{\phi})} r_{S}, \quad r_{L} \rightarrow e^{i(\phi-\bar{\phi})} r_{L}
$$

As a result, the entries of the matrix $\widetilde{\mathcal{L}}$ listed above are manifestly rephasing invariant.

\section{APPENDIX B}

In order to study the time evolution of $B^{0}-\overline{B^{0}}$ observables, one has to solve the evolution equation (3.16),

$$
\frac{d}{d t}|\tilde{\rho}(t)\rangle=\left[\mathcal{H}_{0}+\tilde{\mathcal{L}}\right]|\tilde{\rho}(t)\rangle
$$

with $\widetilde{\mathcal{L}}$ as in $(3.21)$, for a given initial state $|\tilde{\rho}(0)\rangle$. In other terms, one has to compute the entries of the $4 \times 4$ evolution matrix $M_{i j}(t)$, which gives the components $\tilde{\rho}_{1}(t), \tilde{\rho}_{2}(t)$, $\tilde{\rho}_{3}(t), \tilde{\rho}_{4}(t)$ of the state vector $|\tilde{\rho}(t)\rangle$ at time $t$, in terms of the initial ones at $t=0$ :

$$
\tilde{\rho}_{i}(t)=\sum_{j=1}^{4} M_{i j}(t) \tilde{\rho}_{j}(0), \quad i=1,2,3,4
$$


As explained in the text, it is sufficient to write down an approximate expression for $M_{i j}(t)$ that contains contributions up to first order in the dissipative parameters (3.22) appearing in $\widetilde{\mathcal{L}}$. The expansion of $M_{i j}(t)$ within this approximation can be conveniently organized as the sum of two contributions:

$$
\tilde{\rho}_{i}(t) \simeq \sum_{j=1}^{4}\left[M_{i j}^{(0)}(t)+M_{i j}^{(1)}(t)\right] \tilde{\rho}_{j}(0) .
$$

The matrix $M^{(0)}$ has only diagonal non-vanishing terms:

$$
\begin{array}{ll}
M_{11}^{(0)}(t)=e^{-\gamma_{S} t}, & M_{33}^{(0)}(t)=e^{-\left(\Gamma_{-}+\widetilde{A}-\widetilde{D}\right) t}, \\
M_{22}^{(0)}(t)=e^{-\gamma_{L} t}, & M_{44}^{(0)}(t)=e^{-\left(\Gamma_{+}+\widetilde{A}-\widetilde{D}\right) t} .
\end{array}
$$

The entries of $M^{(1)}$ take instead the following explicit expression:

$$
\begin{aligned}
& M_{11}^{(1)}(t)=0 \\
& M_{13}^{(1)}(t)=\frac{2 \widetilde{C}}{\Delta \Gamma_{+}}\left(e^{-\gamma_{S} t}-e^{-\Gamma_{-} t}\right) \\
& M_{12}^{(1)}(t)=\frac{\widetilde{D}}{\Delta \Gamma}\left(e^{-\gamma_{L} t}-e^{-\gamma_{S} t}\right) \\
& M_{14}^{(1)}(t)=\frac{2 \widetilde{C}^{*}}{\Delta \Gamma_{-}}\left(e^{-\gamma_{S} t}-e^{-\Gamma_{+} t}\right) \\
& M_{21}^{(1)}(t)=\frac{\widetilde{D}}{\Delta \Gamma}\left(e^{-\gamma_{L} t}-e^{-\gamma_{S} t}\right) \\
& M_{22}^{(1)}(t)=0 \\
& M_{23}^{(1)}(t)=\frac{2 \widetilde{C}}{\Delta \Gamma_{-}}\left(e^{-\gamma_{L} t}-e^{-\Gamma_{-} t}\right) \\
& M_{24}^{(1)}(t)=\frac{2 \widetilde{C}^{*}}{\Delta \Gamma_{+}}\left(e^{-\gamma_{L} t}-e^{-\Gamma_{+} t}\right) \\
& M_{31}^{(1)}(t)=\frac{2 \widetilde{C}^{*}}{\Delta \Gamma_{+}}\left(e^{-\gamma_{S} t}-e^{-\Gamma_{-} t}\right) \\
& M_{32}^{(1)}(t)=\frac{2 \widetilde{C}^{*}}{\Delta \Gamma_{-}}\left(e^{-\gamma_{L} t}-e^{-\Gamma_{-} t}\right) \\
& M_{33}^{(1)}(t)=0 \\
& M_{34}^{(1)}(t)=\frac{i \widetilde{B}}{2 \Delta m}\left(e^{-\Gamma_{+} t}-e^{-\Gamma_{-} t}\right) \\
& M_{41}^{(1)}(t)=\frac{2 \widetilde{C}}{\Delta \Gamma_{-}}\left(e^{-\gamma_{S} t}-e^{-\Gamma_{+} t}\right) \\
& M_{42}^{(1)}(t)=\frac{2 \widetilde{C}}{\Delta \Gamma_{+}}\left(e^{-\gamma_{L} t}-e^{-\Gamma_{+} t}\right) \\
& M_{43}^{(1)}(t)=\frac{i \widetilde{B}^{*}}{2 \Delta m}\left(e^{-\Gamma_{+} t}-e^{-\Gamma_{-} t}\right) \quad M_{44}^{(1)}(t)=0
\end{aligned}
$$

where $\Delta \Gamma_{ \pm}=\Delta \Gamma \pm 2 i \Delta m$. In presenting the above expressions for the entries of $M^{(0)}$ and $M^{(1)}$, we have reconstructed the exponential dependences out of first order correction terms which are linear in time.[21]

Although the time dependence in the solution of $(B .1)$ is always exponential, this feature is lost in perturbation theory. However, to a given order in the perturbative 
expansion, one can always reconstruct the correct exponential behaviour by a redefinition of the parameters $\gamma_{S}, \gamma_{L}$ and $\Delta m$, so that they coincide with the widths and mass difference of the physical states $\left|B_{S}\right\rangle$ and $\left|B_{L}\right\rangle$. To first order, only the widths get shifted: $\gamma_{S} \rightarrow \gamma_{S}+\widetilde{D}$, $\gamma_{L} \rightarrow \gamma_{L}+\widetilde{D}$. Accordingly, if we redefine $\Gamma$ to be the average of the new $\gamma_{S}$ and $\gamma_{L}$, then the quantities $\Gamma_{ \pm}$get changed as: $\Gamma_{ \pm} \rightarrow \Gamma_{ \pm}+\widetilde{A}-\widetilde{D}$. This explains the form of the exponential terms in (B.4).

\section{APPENDIX C}

As explained in the text, the evolution in time of the two correlated neutral $B$-mesons coming from the decay of the $\Upsilon(4 S)$ resonance can be obtained using the single meson dynamics $|\tilde{\rho}(0)\rangle \rightarrow|\tilde{\rho}(t)\rangle$ generated by the equation (3.16). It results convenient to regroup the four components of the vector $|\tilde{\rho}(t)\rangle$ into a $2 \times 2$ matrix

$$
\tilde{\rho}(t)=\left[\begin{array}{cc}
\tilde{\rho}_{1}(t) & \tilde{\rho}_{3}(t) \\
\tilde{\rho}_{4}(t) & \tilde{\rho}_{2}(t)
\end{array}\right]
$$

it can be expressed in terms of the elementary operators $\widetilde{P}_{i}, i=1,2,3,4$, introduced in $(6.5)$ :

$$
\tilde{\rho}(t) \equiv \sum_{i=1}^{4} \widetilde{P}_{i} \tilde{\rho}_{i}(t) .
$$

In order to compute explicitly the form of the various observables involving correlated mesons, we need to determine the time evolution of the operators $\widetilde{P}_{i}$. Using the results of Appendix B, on easily finds [adopting the same notation as in (C.1)]:

$$
\begin{array}{ll}
\widetilde{P}_{1}(t)=\left[\begin{array}{ll}
M_{11}^{(0)}(t) & M_{31}^{(1)}(t) \\
M_{41}^{(1)}(t) & M_{21}^{(1)}(t)
\end{array}\right] & \widetilde{P}_{2}(t)=\left[\begin{array}{ll}
M_{12}^{(1)}(t) & M_{32}^{(1)}(t) \\
M_{42}^{(1)}(t) & M_{22}^{(0)}(t)
\end{array}\right] \\
\widetilde{P}_{3}(t)=\left[\begin{array}{ll}
M_{13}^{(1)}(t) & M_{33}^{(0)}(t) \\
M_{43}^{(1)}(t) & M_{23}^{(1)}(t)
\end{array}\right] & \widetilde{P}_{4}(t)=\left[\begin{array}{ll}
M_{14}^{(1)}(t) & M_{34}^{(1)}(t) \\
M_{44}^{(0)}(t) & M_{24}^{(1)}(t)
\end{array}\right] .
\end{array}
$$

With these expressions, on can now calculate the elementary double probabilities in (6.10)

$$
\begin{aligned}
\mathcal{P}_{i j}\left(t_{1}, t_{2}\right)=\operatorname{Tr}\left\{\widetilde{P}_{i} \widetilde{P}_{1}\left(t_{1}\right)\right\} \operatorname{Tr}\left\{\widetilde{P}_{j} \widetilde{P}_{2}\left(t_{2}\right)\right\}+\operatorname{Tr}\left\{\widetilde{P}_{i} \widetilde{P}_{2}\left(t_{1}\right)\right\} \operatorname{Tr}\left\{\widetilde{P}_{j} \widetilde{P}_{1}\left(t_{2}\right)\right\} \\
-\operatorname{Tr}\left\{\widetilde{P}_{i} \widetilde{P}_{3}\left(t_{1}\right)\right\} \operatorname{Tr}\left\{\widetilde{P}_{j} \widetilde{P}_{4}\left(t_{2}\right)\right\}-\operatorname{Tr}\left\{\widetilde{P}_{i} \widetilde{P}_{4}\left(t_{1}\right)\right\} \operatorname{Tr}\left\{\widetilde{P}_{j} \widetilde{P}_{3}\left(t_{2}\right)\right\},
\end{aligned}
$$

and, with a further time integration, the single-time probabilities

$$
\Pi_{i j}(t)=\int_{0}^{\infty} d t^{\prime} \mathcal{P}_{i j}\left(t^{\prime}+t, t^{\prime}\right)
$$

Introducing again the variable $\tau=\Gamma t$ and recalling the definitions (5.3) and (5.6), one explicitly finds: 


$$
\begin{aligned}
& \Pi_{11}(\tau)=\frac{e^{-\tau}}{2 \Gamma} \frac{D}{(1+\delta)}\left[\cosh \delta \tau+\frac{\sinh \delta \tau}{\delta}\right] \\
& \Pi_{12}(\tau)=\frac{e^{-\tau}}{2 \Gamma} e^{-\delta \tau}, \\
& \Pi_{13}(\tau)=\frac{e^{-\tau}}{2 \Gamma} \frac{C}{\delta+i \omega}\left[e^{i \omega \tau}-r^{*} e^{-\delta \tau}\right], \\
& \Pi_{14}(\tau)=\left[\Pi_{13}(\tau)\right]^{*}, \\
& \Pi_{21}(\tau)=\frac{e^{-\tau}}{2 \Gamma} e^{\delta \tau} \\
& \Pi_{22}(\tau)=\frac{e^{-\tau}}{2 \Gamma} \frac{D}{(1-\delta)}\left[\cosh \delta \tau+\frac{\sinh \delta \tau}{\delta}\right], \\
& \Pi_{23}(\tau)=\frac{e^{-\tau}}{2 \Gamma} \frac{C}{\delta-i \omega}\left[e^{i \omega \tau}-\frac{e^{\delta \tau}}{r}\right] \\
& \Pi_{24}(\tau)=\left[\Pi_{23}(\tau)\right]^{*} \text {, } \\
& \Pi_{31}(\tau)=\frac{e^{-\tau}}{2 \Gamma} \frac{C}{\delta+i \omega}\left[e^{\delta \tau}-r^{*} e^{-i \omega \tau}\right] \\
& \Pi_{32}(\tau)=\frac{e^{-\tau}}{2 \Gamma} \frac{C}{\delta-i \omega}\left[e^{-\delta \tau}-\frac{e^{-i \omega \tau}}{r}\right], \\
& \Pi_{33}(\tau)=-\frac{e^{-\tau}}{2 \Gamma} \frac{B^{*}}{1+i \omega}[\omega \cos \omega \tau+\sin \omega \tau] \text {, } \\
& \Pi_{34}(\tau)=-\frac{e^{-\tau}}{2 \Gamma} \frac{e^{-(A-D+i \omega) \tau}}{1+A-D}, \\
& \Pi_{41}(\tau)=\left[\Pi_{31}(\tau)\right]^{*}, \\
& \Pi_{42}(\tau)=\left[\Pi_{32}(\tau)\right]^{*}, \\
& \Pi_{43}(\tau)=\left[\Pi_{34}(\tau)\right]^{*}, \\
& \Pi_{44}(\tau)=\left[\Pi_{33}(\tau)\right]^{*},
\end{aligned}
$$

where the parameter $r$ represents the following ratio:

$$
r=\frac{2-\delta+i \omega}{2+\delta-i \omega}
$$

These expressions for the components of $\Pi_{i j}(\tau)$ have been used to compute the double decay rates $\Gamma\left(f_{1}, f_{2} ; \tau\right)$ in $(6.11)$. 


\section{REFERENCES}

1. G.C. Branco, L. Lavoura and J.P. Silva, CP Violation, (Clarendon Press, Oxford, 1999)

2. I.I. Bigi and A.I. Silva, CP Violation, (Cambridge Univeristy Press, Cambridge, 2000)

3. P. Eerola, Nucl. Instr. and Meth. A446 (2000) 384

4. R. Alicki and K. Lendi, Quantum Dynamical Semigroups and Applications, Lect. Notes Phys. 286, (Springer-Verlag, Berlin, 1987)

5. V. Gorini, A. Frigerio, M. Verri, A. Kossakowski and E.C.G. Surdarshan, Rep. Math. Phys. 13 (1978) 149

6. H. Spohn, Rev. Mod. Phys. 52 (1980) 569

7. A. Royer, Phys. Rev. Lett. 77 (1996) 3272

8. C.W. Gardiner and P. Zoller, Quantum Noise, 2nd. ed. (Springer, Berlin, 2000)

9. W.H. Louisell, Quantum Statistical Properties of Radiation, (Wiley, New York, 1973)

10. M.O. Scully and M.S. Zubairy, Quantum Optics (Cambridge University Press, Cambridge, 1997)

11. L. Fonda, G.C. Ghirardi and A. Rimini, Rep. Prog. Phys. 41 (1978) 587

12. H. Nakazato, M. Namiki and S. Pascazio, Int. J. Mod. Phys. B10 (1996) 247

13. F. Benatti and R. Floreanini, Phys. Lett. B428 (1998) 149

14. F. Benatti and R. Floreanini, Phys. Lett. B451 (1999) 422

15. F. Benatti and R. Floreanini, JHEP 02 (2000) 032

16. F. Benatti and R. Floreanini, Phys. Rev. D 62 (2000) 125009

17. A.D. Dolgov, Sov. J. Nucl. Phys. 33 (1981) 700

18. G. Sigl and G. Raffelt, Nucl. Phys. B406 (1993) 423

19. J. Ellis, J.L. Lopez, N.E. Mavromatos and D.V. Nanopoulos, Phys. Rev. D 53 (1996) 3846

20. P. Huet and M.E. Peskin, Nucl. Phys. B434 (1995) 3

21. F. Benatti and R. Floreanini, Nucl. Phys. B488 (1997) 335

22. F. Benatti and R. Floreanini, Phys. Lett. B401 (1997) 337

23. F. Benatti and R. Floreanini, Nucl. Phys. B511 (1998) 550

24. F. Benatti and R. Floreanini, CPT, dissipation, and all that, in Physics and Detectors for Daphne, "Frascati Physics Series", vol. XVI, 1999, p. 307, hep-ph/9912426

25. F. Benatti and R. Floreanini, Phys. Lett. B465 (1999) 260

26. M.S. Marinov, JETP Lett. 15 (1972) 479; Sov. J. Nucl. Phys. 19 (1974) 173; Nucl. Phys. B253 (1985) 609

27. S. Hawking, Comm. Math. Phys. 87 (1983) 395; Phys. Rev. D 37 (1988) 904; Phys. Rev. D 53 (1996) 3099; S. Hawking and C. Hunter, Phys. Rev. D 59 (1999) 044025 
28. J. Ellis, J.S. Hagelin, D.V. Nanopoulos and M. Srednicki, Nucl. Phys. B241 (1984) 381

29. S. Coleman, Nucl. Phys. B307 (1988) 867

30. S.B. Giddings and A. Strominger, Nucl. Phys. B307 (1988) 854

31. M. Srednicki, Nucl. Phys. B410 (1993) 143

32. W.G. Unruh and R.M. Wald, Phys. Rev. D 52 (1995) 2176

33. L.J. Garay, Phys. Rev. Lett. 80 (1998) 2508; Phys. Rev. D 58 (1998) 124015

34. J. Ellis, N.E. Mavromatos and D.V. Nanopoulos, Phys. Lett. B293 (1992) 37; Int. J. Mod. Phys. A11 (1996) 1489

35. F. Benatti and R. Floreanini, Ann. of Phys. 273 (1999) 58

36. V.A. Kostelecký and R. Van Kooten, Phys. Rev. D 54 (1996) 5585

37. P. Colangelo and G. Corcella, Eur. Phys. J. C 1 (1998) 515

38. S. Yang and G. Isidori, Test of $C P T$ invariance in semileptonic $B$ decays, BaBar Note \#438, 1998

39. R.A. Bertlmann and W. Grimus, Phys. Rev. D 58 (1998) 034014

40. A. Mohapatra, M. Satpathy, K. Abe and Y. Sakai, Phys. Rev. D 58 (1998) 036003

41. L. Lavoura and J.P. Silva, Phys. Rev. D 60 (1999) 056003

42. F. Benatti and R. Floreanini, Mod. Phys. Lett. A12 (1997) 1465; Banach Center Publications, 43 (1998) 71; Phys. Lett. B468 (1999) 287; On the weak-coupling limit and complete positivity, Chaos Sol. Frac., to appear

43. F. Benatti and R. Floreanini, J. Phys. A33 (2000) 8139

44. N.W. Tanner and R.H. Dalitz, Ann. of Phys. 171 (1986) 463

45. M.C. Banuls and J. Bernabeu, Phys. Lett. B423 (1998) 151; B464 (1999) 117; Nucl Phys. B590 (2000) 19

46. L. Lavoura, Phys. Lett. B442 (1998) 390

47. The OPAL Collaboration, Zeit. fur Physik C76 (1997) 401

48. The OPAL Collaboration, Eur. Phys. J. C12 (2000) 609

49. P. Ball et al., $B$ decays at the LHC, CERN-TH-2000-101, hep-ph/0003238

50. The BTeV proposal, 2000, http://www-btev.fnal.gov

51. The BaBar Collaboration, The BaBar Physics Book, SLAC-R-504, 1998

52. G. De Domenico and Ch. Yèche, Dilepton analysis in BaBar experiment: measurement of the mixing parameter $\Delta m_{B}$ and study of the $T(C P)$ violation purely in mixing, BaBar Note \#409, 1998

53. C. Leonidopoulos, $B^{0}-\overline{B^{0}}$ mixing and $C P T$ violation with the Belle experiment, Ph.D. thesis, Princeton University, 2000

54. The CLEO Collaboration, Phys. Lett. B490 (2000) 37

55. Particle Data Group, Eur. Phys. J. C 15 (2000) 1

56. S. Adler, Phys. Rev. D 62 (2000) 117901

57. J. Ellis, N.E. Mavromatos and D.V. Nanopoulos, Phys. Rev. D 63 (2001) 024024 